Roxana Iordache

Bucureşti

\title{
REMARQUES SUR LA SUBORDONNÉE TEMPORELLE A L'ÉPOQUE CLASSIQUE ET A L'ÉPOQUE TARDIVE, CHEZ JORDANES $-2^{\mathrm{e}}$ partie
}

(Voir: Linguistica XXXII, pags. 31-60)

\section{LE RAPPORT D'ANTÉRIORITÉ}

Le latin cultivé de l'époque classique marquait l'antériorité par une multitude de conjonctions et de locutions conjonctives, construites avec certains temps de l'indicatif (parfois avec le subjonctif aussi); ces conjonctions et locutions conjonctives sont plus nombreuses que celles qui servaient à exprimer le rapport de simultanéité, ou de postériorité.

On distingue, dans le cadre du rapport d'antériorité:

A) une antériorité récente (immédiate) par rapport à l'action de la principale (sens: "dès que" et "quand", "lorsque"),

B) une antériorité plus accentuée, ou non-déterminée dans le temps (sens "après que");

C) le moment où l'action de la principale commence (sens "depuis que").

A) Le sens "dès que", "lorsque", était rendu par les conjonctions et les locutions suivantes: ut et ut primum, ubi et ubi primum, cum "uere temporale", simul ac, simul atque (ainsi que les formes simulac, simulatque), simul. Peu souvent, les grands écrivains de l'époque classique emploient simul et, simulac primum, simul ut. ${ }^{137}$ Parfois, le sens "dès que", "lorsque", était rendu par cum narratif, suivi du subjonctif plus-queparfait.

$V t$ et ut primum sont construits, en général, avec le parfait historique; rarement apparaissent le présent historique et le futur; on rencontre assez fréquemment le futur antérieur; isolément, $u t$ et $u t$ primum sont suivis de l'indicatif imparfait et plus-queparfait (l'imparfait est employé, d'habitude, dans des propositions temporelles exprimant un rapport de simultanéité, ou dans des subordonnées explicatives causales indiquant une légère antériorité de la protase par rapport à l'apodose).

137 Pour ce qui est de la rareté et la construction de ces locutions conjonctives, voir, notamment, R. Kühner C. Stegmann, Ausfihrliche Grammatik der lateinischen Sprache, II - op. cit., p. $359^{4}$, p. 364 , n 10 , $a$ et b. 
Vbi et ubi primum sont construits le plus souvent avec le parfait historique, moins fréquemment avec le présent historique et le futur; le futur antérieur est relativement fréquent; l'imparfait est employé isolément (même remarque que dans le cas de $u t$ ); le plus-que-parfait de l'indicatif est rare.

Cum temporel proprement dit est suivi surtout du parfait historique et - moins souvent - du présent historique et de l'imparfait (pour ce dernier - même emploi que dans le cas de $u t$ et $u b i$ ); le futur et le futur antérieur apparaissent plus rarement aussi.

Simul ac, simul atque et simul requièrent en général l'indicatif, parfois aussi l'imparfait, le futur et le futur antérieur.

B) Le sens "après que" était exprimé par cum narratif et postquam. Cum narratif est construit avec le subjonctif plus-que-parfait. Postquam apparaît suivi en général du parfait historique, assez souvent du présent historique et rarement du plus-que-parfait (équivalant à un imparfait d'état) et de l'imparfait de l'indicatif. Construit avec l'imparfait de l'indicatif, postquam a, en général, une nuance causale.

C) Pour le sens "depuis que", on utilisait, dans le latin cultivé de l'époque classique: ut (avec le parfait logique et le véritable présent), cum (avec le parfait et le présent), postquam (d'habitude: post .... quam), suivi du perfectum praesens, du véritable présent et du plus-que-parfait de l'indicatif; peu souvent on employait quod (avec le présent et le parfait). Quam n'apparaissait que rarement sans l'antécédant post dans la principale.

Quam pouvait aussi indiquer l'antériorité de la subordonnée dans des contextes comme: "non prius sum conatus misericordiam aliis commouere, quam misericordia sum ipse captus.", Cicéron, De or., 2, 195. Dans de telles situations, on emploie, d'habitude, dans la subordonnée, le parfait (de l'indicatif) et le futur antérieur.

Les temporelles dont l'action est antérieure à l'action de la principale sont, chez Jordanès, introduites par une multitude de conjonctions.

Tant les propositions exprimant l'antériorité immédiate, que celles indiquant l'antériorité non déterminée sont fréquentes chez Jordanès. Les subordonnées marquant le moment où commence l'action de la régissante sont rares.

Les conjonctions qui, chez Jordanès, marquent l' antériorité immédiate sont: $u b i$, mox, ut, cum narratif, dum construit avec le subjonctif plus-que-parfait, cum "uere temporale" (pour ce dernier, voir le commentaire pages 50-51 de la première partie de cette étude) postquam, la locution conjonctive cum mox et, enfin, quod - l'énumération a été faite par ordre décroissant de leur fréquence dans le texte.

Simul ac, simul atque et simul, en régression dès le latin classique, n'apparaissent jamais chez Jordanès, encore moins les locutions simul et et simul primum.

Les conjonctions indiquant une plus grande distance temporelle entre la subordonnée et la principale, ou bien une antériorité non déterminée sont, chez Jordanès: 
cum narratif, postquam et dum - énumérés toujours par ordre décroissant de leur fréquence.

Le moment où commence l'action dans la principale est marquée par quam et postquam.

Le rapport d'antériorité est indiqué aussi chez Jordanès par: "non prius + Verbe principal - quam" ou "non ante + Verbe principal - nisi" (voir le commentaire supra).

A) la plus fréquente conjonction temporelle indiquant l'antériorité immédiate $\mathrm{ubi}^{138}$ n'apparaît, pourtant, que onze fois avec cette valeur. A cela s'ajoutent quatre exemples où ubi indique un rapport temporel de simultanéité (ou à mi-chemin entre la simultanéité et l'antériorité), sans compter les quatres exemples de ubi itératif(voir le paragraphe sur les "Propositions temporelles de répétition") et les deux exemples de ubi explicatif-causal.

On peut donc tirer la conclusion que Jordanès emploie $u b i$ notamment pour indiquer l'antériorité immédiate. Voici la répartition de ubi marquant l'antériorité, dans les deux ouvrages de Jordanès (sans compter les exemples de ubi itératif et de ubi explicatif-causal): cinq exemples dans les Romana et six dans les Getica.

Vbi temporel pour des actions non répétées indique donc, chez Jordanès:

1. un rapport de simultanéité, voir Romana, 131: "Tunc Clusium Tusciae urbem obsidebant, ubi pro sociis ac foederatis Romanus interuenit missis ex more legatis." (construction différente chez Florus, 1, 7, 6: "Tum Clusium urbem obsidebant. Pro sociis ac foederatis Romanus interuenit; missi ex more legati.") Des exemples du même genre apparaissent dans les Getica, 110 et Romana, 121.

2. un rapport situé à mi-chemin entre la simultanéité et l'antériorité, voir Getica, 46: "Sed ubi fit Ponto uicinior, paruum fontem suscipit, cui Exampheo cognomen est..." (le passage est inspiré de Pomp. Méla, 2, 1, 7, mais la construction grammaticale appartient à Jordanès);

3. un rapport d'antériorité immédiate: "Quod ubi desperauit (Cleopatra) a principe seruari que ${ }^{139}$ se triumpho cognouit,... in mausuleum ${ }^{140}$ se regum recepit ibique. ... iuxta suum se conlocauit ${ }^{141}$ Antonium admotisque ad uenas serpentibus, sic morte quasi somno soluta est.", Rom., 254 (voir Florus, 2, 21, 10-11).

138 Vbi apparaît fréquemment chez Jordanès en tant que adverbe et conjonction introduisant des propositions relatives. C'est un mot que le latin vulgaire semble préférer; il s'est transmis dans toutes les langues romanes (voir Wilh. Meyer-Lübke, R.E.W., op. cit., n 9028) avec son sens local et même temporel (voir Wilh. Meyer-Lübke, Grammatik der romanischen Sprachen - Romanische Syntax, vol. III, Leipzig, 1899, par. 514), dans ses propositions interrogatives et relatives.

139 Infinitif présent au lieu de l'infinitiffutur; c'est toujours l'infinitif présent qui apparâit, pour la même idée, chez Florus, 2, 21, 10-11. Pour d'autres exemples de ce type, chez Jordanès, voir note 50.

140 mausuleum pour mausoleum. Sur la confusion entre o et $u$, voir les notes antérieures.

141 conlocauit pour collocauit (dans le passage de Florus - que, d'ailleurs, Jordanès copie - c'est toujours conlocauit que nous trouvons). Voir aussi Getica, 197: conlocantes. Voir également les notes suivantes. 
Quatre de cinq exemples des Romana indiquant l'antériorité sont entièrement copiés sur Florus, voir l'exemple déjà cité et, en plus, Romana, 97, 184 et 254.

Parmi les six exemples des Getica, un seul est à coup sûr inspiré d'un autre auteur - voir Getica, 85 (même construction grammaticale dans l'Histoire Auguste, Vita Maximini, 3).

Vbi exprimant l'antériorité est construit, généralement, avec le parfait de l'indicatif (voir Romana, 184, 252, 254; Getica, 85, 140, 142, 195, 218), parfois avec le présent historique (Romana, 97, 381). Dans la proposition corrélative, on trouve, dans la plupart des cas, l'indicatif parfait et, assez souvent (dans cinq cas) - le présent historique. Le présent historique est, chez Jordanès, plus fréquent dans les principales que dans les temporelles d'antériorité, ce qui correspond d'ailleurs à l'esprit du latin cultivé de l'époque classique (on met en évidence, de la sorte, l'antériorité de la subordonnée temporelle - voir, pour comparer, César, B.G., 1, 7, 3 etc.).

Ce qui est intéressant, c'est l'emploi du subjonctif après $u b i$ : "Quod $u b i$ rex Hunnorum Dintzic filius Attilae cognouisset, collectis secum qui adhuc uidebantur quamuis pauci eius tamen sub imperio remansisse, Vltzinzures, Angisciros, Bittugures, Bardores $^{142}$, uenientesque ad Basianam ${ }^{143}$ Pannoniae ciuitatem eamque circumuallans fines eius coepit praedare $144 . "$, Get., 272. Voici la traduction de ce fragment: "Dès que Dintzic, roi des Huns, fils d'Attila, apprit cela, réunissant autour de lui ceux qui, quoique peu nombreux, semblaient être restés sous son commandement, à savoir les Ultzinzures, les Angiscires, les Bittugures, les Bardores, et marchant tous sur Bassiana, cité de la Pannonie, il l'assiégea et commença à saccager son territoire." Cfr. Romana, 254: "ubi. ... cognouit ..." (exemple déjà cité); cfr. Getica, 260: "ut ... conperit...".

L'emploi du subjonctif à la place de l'indicatif après $u b i$ semble avoir été un phénomène spécifique de la langue parlée. ${ }^{145}$ Pour des actions non répétées, le subjonctif est attesté, pour la première fois, dans le Bellum Africum, 78, 4 (en alternance avec l'indicatif), ensuite chez Tite-Live, 26, 11, 3, Tacite, Hist., 2, 40 et surtout en latin tardif - voir Ammien, 25, 4, 5, Rufin, Hist. monach., 29, p. 454 c, Victor de Vite, Hist. persec. Afric. prou., 3, 47 etc. etc.

L'apparition du subjonctif après ubi pourrait s'expliquer par l'influence de la construction de cum narratif de simultanéité et d'antériorité; d'ailleurs, durant toute la

142 Vltzinzures, Angisciros etc. - sujets en accusatif d' une Participiale absolue dont le verbe se trouve à l'Ablatif. De telles situations réapparaissent chez Jordanès (voir Romana, 331; ibid., 366, 369 etc.).

143 Basianam pour Bassianam. On remarque, de plus, l' emploi de la préposition avant un nom de ville marquant la direction.

144 Infinitif actif d'un verbe déponent (les formes actives incorrectes sont plus fréquentes pour son composé depraedari, voir les notes antérieures). La forme correcte de cet infinitif présent (praedari) apparaît dans le même paragraphe, une ligne plus haut (Get., 272).

145 Al. Ernout et Fr. Thomas, op. cit., par. 362 (pag. 367), affirment: "César, par un scrupule de puriste, évitait, même au style indirect, d'employer $u b i$ avec un imparfait ou un plus-que-parfait du subjonctif." 
latinité, seuls, du subjonctif, l'imparfait et le plus-que-parfait semblent avoir été employés après $u b i$, pour exprimer des actions non répétées. ${ }^{146} \mathrm{Chez}$ Jordanès, l'unique exemple construit avec le subjonctif - Getica, 272 - apparaît avec le plus-que-parfait (Cfr. Jordanès, Getica, 141: “quod cum ... conperisset...”).

On ne saurait exclure comme explication non plus l'influence qu'exerce dum, si fréquemment construit (en bas latin) avec l'imparfait du subjonctif. A cela s'ajoutait, vraisemblablement, l'influence de la construction de postquam, fréquemment suivi du subjonctif en latin tardif (voir ci-dessous).

Rappelons aussi la perte de la valeur modale (volitive ou potentielle) du subjonctif plus-que-parfait.

Nous devons souligner que l'emploi du subjonctif plus-que-parfait après ubi non itératif est rare tout le long de la latinité ${ }^{147}$. Ajoutons que l'unique exemple de ce genre chez Jordanès se trouve dans les Getica.

Vt temporel n'apparaît que rarement chez Jordanès: dix exemples auxquels s'ajoute un exemple de $u t$ temporel-causal et plusieurs exemples de $u t$ causal. Rappelons que, chez Jordanès, il y a un seul exemple de ut itératif.

Sa fréquence faible correspond à une tendance, de longue date déjà en latin, à remplacer d'abord le $u t$ temporel (tant au sens de: "quand", "dès que", que dans l'acception de "depuis que", mais surtout dans les premières acceptions - qui, d'ailleurs, étaient plus fréquentes aussi ${ }^{148}$ ), et puis à substituer $u t$ dans les autres fonctions aussi (les dernières valeurs qui disparaissaient étant celles construites avec le subjonctif. ${ }^{149}$ )

Donc, conformément à l'esprit du bas latin, ut temporel est rare chez Jordanès, un peu plus rare que $u b i$.

$V t$ temporel indique en général, chez Jordanès, l'antériorité immédiate et, dans cette qualité, il est toujours suivi de l'indicatif. Voir Getica, 260: “Quod ut Gepidarum rex conperit ${ }^{150}$ Ardarichus, ... contra ${ }^{151}$ filios Attilae primus insurgit inlatumque ${ }^{152}$

146 Voir Al. Emout - Fr. Thomas, op. cit., p. 367; J. B. Hofmann - A. Szantyr, 2. Teil, 2. Bd., op. cit., p. 652.

147 Voir J. B. Hofmann - A. Szantyr, II -2, op. cit., p. 652.

148 Déjà chez Térence, $u t$ avec le sens de "lorsque" est remplacé par la conjonction cum. A l'époque classique, $u t$ temporel avec le sens de "lorsque" et "dès que" est rarement utilisé et cette tendance s'accrô̂t en latin impérial et au commencement du Moyen Age. Dans l'entière oeuvre d'Avit (fin du V $\mathrm{V}^{\mathrm{e}}$ siècle et commencement du $\mathrm{VI}^{\mathrm{e}}$ siècle), ut temporel apparaît quelques fois seulement (voir $\mathrm{H}$. Goelzer, Le latin de Saint Avit, op. cit., par. 218).

$V t$ causal, provenant du sens temporel "dès que", est très rare chez Jordanès et chez d'autres auteurs tardifs. Voir aussi la note suivante.

149 Quant aux valeurs “subjonctives” de $u t$, voir Al. Ernout - Fr. Thomas, op. cit., pp. 294 et $360 . V t$ s'est conservé en très faible mesure dans les langues romanes (voir calabr.: uti, utu; vénit.: ute, uti - voir Wilh. Meyer-Lübke, R. E. W., op. cit., 9099, a).

150 conperit pour comperit, apparait souvent chez Jordanès (voir Romana, 118: conperisset; ibid., 333: conperit ; 376: conperiens; Get., 137: conperiens; ibid., 141: conperisset etc.). Voir également la note 75 à propos de conpraehenderent.

151 Remarquer la grande fréquence de la préposition contra (voir notes 98 et 113). 
seruiendi pudore ${ }^{153}$ secuta felicitate detersit...”. Voir également Romana, 245 et 363 exemples différant de leurs modèles au point de vue de la construction grammaticale; voir aussi Getica, 25, 105, 111, 129,.

En général, le temps des subordonnées d'antériorité est le parfait de l'indicatif, rarement - le présent historique. Dans les principales, on rencontre d'habitude le parfait de l'indicatif et, rarement, le présent historique; dans une phrase, on assiste à l'alternance - dans les principales - du présent historique et du parfait (historique) de l'indicatif, pour la même subordonnée - voir l'exemple cité, Getica, 260.

Chez Jordanès, ut temporel indique rarement la simultanéité. Voir Getica, 70: "Qualis erat, rogo, uoluptas, $u t$ uiri fortissimi, quando ab armis quantolumcumque ${ }^{154}$ uacassent, doctrinis philosophicis inbuebantur ${ }^{155}$ ?"

L'autre exemple de ut temporel marquant la simultanéité se trouve toujours dans les Getica, mais, cette fois-ci, il est construit avec l'imparfait du subjonctif: "Nam ${ }^{156}$ et ut famosissmam et Romae emulam ${ }^{157}$ in suo nomine ${ }^{158}$ conderet ciuitatem, Gothorum interfuit operatio, qui foedus ${ }^{159}$ inito cum imperatore quadraginta suorum milia illi in solacio ${ }^{160}$ contra $^{161}$ gentes uarias obtulere.", Get., 112. Voici la traduction de ce passage: "Et, pendant que Constantin construisait cette plus que fameuse cité, rivale de Rome, à laquelle il donna son nom, il fut aidé par les Goths qui, concluant une alliance avec l'empereur, lui offrirent, comme secours contre les diverses peuplades, quarante mille soldats des leurs".

L'emploi du subjonctif après $u t$, lorsqu'il ne s'agit ni de répétition, ni de style indirect, ni d'attraction modale est un fait du latin familier et vulgaire. Le premier exemple de ce type semble être attesté dans le Pseudo Quintilien, Decl., 330, p. 299, 5, ensuite dans les Carmina epigraphica, 1590, 6, Panegyrici, 9 (12), 26, 5, Peregrinatio Aetheriae, 3, 6, etc. ${ }^{162}$

152 inlatum pour illatum (voir aussi inpatiens - Romana, 380; inposita - Romana, 133; inportunitatem Rom., 107 etc.). Les mots composés à l'aide du préfixe reconstitué (ou conservé) - le préfixe in- ou un autre - sous sa forme primitive sont fréquents chez Jordanès. Voir aussi notes 127 et 133.

153 forme d'accusatif où la finale $-m$ n'est pas marquée (voir cependant inlatum).

154 Pour cet adverbe, voir note 126.

155 Pour le maintien de la sonnante $-n$ dans le préfixe in-, voir note 127.

156 nam - superflu.

157 Emulam au lieu de aemulam.

158 complément à préposition à la place d'un ablatif sans préposition.

159 foedus - sujet en accusatif dans une construction "participium absolutum". Le participe - prédicat (inito) se trouve cependant à l'ablatif. Voir aussi note 142.

160 in solacio pour solacio, ablatif prépositionnel à la place du datif final.

161 Voir note 151.

162 Pour d'autres exemples appartenant à l'époque tardive, voir E. Löfstedt, Beiträge zur Kenntnis des späteren Latein, Diss., Stockholm, 1907, p. 1 sqq. 
C'est sous l'influence de cum narratif que $u t$ commença à être suivi du subjonctif. On peut aussi présumer, pour l'époque tardive, l'influence de $d u m$, presque toujours accompagné de l'imparfait du subjonctif. D'ailleurs, pour le fragment que nous venons de citer (Get., 112), certains manuscrits donnent $d u m$ à la place de $u t .{ }^{163}$

Dans le cas de ut construit avec le subjonctif, le temps usuel était, en latin tardif, l'imparfait; le présent, le plus-que-parfait et le parfait étaient rares. ${ }^{164}$

Il est intéressant de remarquer que l'unique exemple de ubi temporel avec le subjonctif est construit, chez Jordanès, avec le plus-que-parfait, tandis que le seul exemple de ut temporel y apparaît avec l'imparfait du subjonctif (bien que, dans les écrits des auteurs tardifs, y compris chez Jordanès, la tendance fût à remplacer le subjonctif imparfait par le subjonctif plus-que-parfait - voir la discussion supra et note 129). C'est que $u b i$ - plus fréquent, dans ses acceptions temporelles, chez Jordanès, que ut temporel - a des emplois plus diversifiés et plus osés aussi.

Rappelons que l'exemple de $u b i$ temporel suivi du subjonctif se trouve, de même que l'exemple de ut temporel suivi du subjonctif, dans les Getica.

Tant $u b i$ que $u t$ sont parfois accompagnés d'adverbes: c'est un procédé très ancien et de source vulgaire ${ }^{165}$, qui avait comme but de consolider la forme et de préciser le sens de mots à volume réduit, dépourvus de prestance phonétique. De toutes les locutions existant à l'époque classique, Jordanès n'emploie que ubi semel (Romana, 184) et ut primum (Getica, 25). On rencontre aussi, chez Jordanès, la locution plus récente, attestée seulement à partir de l'époque d'Auguste - mox ubi. Le passage qui, chez Jordanès, comprend la locution mox ubi est, en général, inspiré de Florus; mais la locution mox ubi appartient à Jordanès. Voir Jordanès, Romana, 252: "Sed mox ubi ad proelium uintum $^{166}$ est..., prima dux fugae regina cum aurea puppe ueloque purporeo $^{167}$ in altum dedit. Mox secutus Antonius; sed instare uestigiis Caesar." Cfr. Florus, 2, 21, 4 et 8: "Ad primam nouorum motuum famam Caesar a Brundisio traiecerat, ut uenienti bello occurreret; ... Prima dux fugae regina, cum aurea puppe ueloque purpureo, in altum dedit. Mox secutus Antonius, sed instare uestigiis Caesar.".

De tous les corrélatifs possibles pour les conjonctions marquant l'antériorité immédiate, seuls les adverbes mox, statim et ilico apparaissent. Mox apparaît deux fois

163 Voir Codex Breslauiensis, $\mathrm{XI}^{\mathrm{e}}$ siècle, que plusieurs éditions suivent (par exemple: l'édition de M. Savagner, Paris, Garnier, 1924, p. 224; l'édition de G. Fournier de Moujan et M. Nisard, Paris, Firmin-Didot et $C^{\text {ie }}$, 1885 , p. 443 etc.).

164 Sur l'utilisation des temps, voir A. Ernout - Fr. Thomas, op. cit., p. 367; J. B. Hofmann - A. Szantyr, II 2. op. cit., p. 636.

165 Voir E. Löfstedt, Syntactica. Studien und Beiträge zur historischen Syntax des Lateins, II, Lund, 1933, p. 219; J. B. Hofmann, Lateinische Umgangssprache, Heidelberg, 1936, 2. Aufl., p. 97; A. H. Salonius, Vitae Patrum, op. cit., p. 334 etc.

166 Uintum pout uentum. Relativement à la confusion entre $i$ et $e$, voir note 46.

167 purporeo pour purpureo. Relativement à la confusion entre $o$ et $u$, voir les notes 99,121 etc. 
(une fois pour $u b i-R o m a n a, 121$ et une autre sois pour $u t-R o m a n a, 363$ ) et statim et ilico - une seule fois.

Pour ubi de simultanéité on rencontre le corrélatif tunc - un seul exemple (voir l'exemple cité - Romana, 131).

A noter que les adverbes qui servent à préciser le sens temporel des conjonctions $u t$ et $u b i$ sont parfois employés tant dans les subordonnées temporelles (formant des locutions conjonctives), que dans les propositions corrélatives. Voir Getica, 25: “... qui ut primum e nauibus ${ }^{168}$ exientes terras attigerunt, ilico nomen loci ${ }^{169}$ dederunt."; voir également Romana, 184: "Igitur ubi semel se in Spaniam 170 mouit illa grauis et luctuosa Punici belli uis atque tempestas..., statim quodam impetu rapta ${ }^{171}$ medias perfraegit ${ }^{172}$ Alpes..." (texte copié sur Florus, 1, 22, 9).

L'agglomération de marques visant à préciser le sens temporel de la conjonction est un trait caractéristique du latin familier et vulgaire.

On peut observer que l'adverbe mox n'apparait pas dans de telles agglomérations (relativement à l'adverbe mox, voir le commentaire ci-dessous, qui accompagne l'exposé sur la conjonction mox). La distribution des corrélatifs qui soulignent le sens des locutions conjonctives offre des aspects intéressants: ilico renforce le sens de la locution ut primum, tandis que statim met en relief la locution ubi semel.

Quoi qu'il en soit, les adverbes qui soulignent le sens de $u b i$ et $u t$, soit dans le cadre des locutions conjonctives, soit en tant que corrélatifs, sont plutôt rares.

On constate que, par rapport au nombre des exemples, $u t$ d'antériorité est plus souvent précisé par des adverbes que ne l'est $u b i$ d'antériorité; c'est un fait significatif.

L'antériorité récente peut être également marquée par la locution cum nox. Cette combinaison que l'usage classique ignore, équivaut aux locutions cum primum ou ut primum, généralement suivies de l'indicatif parfait. Chez Jordanès, la locution cum mox apparait dans une seule phrase, voir Romana, 178: "Cum mox caluere pugna, statim in sudorem eunt et leui motu quasi sole laxantur". Chose notable, cette phrase est presque entièrement copiée sur Florus (voir 2, 4, "quam mox ${ }^{173}$ caluere pugna, statim in sudorem eunt et leui motu quasi sole laxantur."), à cette différence près que Jordanès remplace quam mox par une autre locution, toujours de la langue vulgaire et, certainement, plus facile à comprendre - cum mox. ${ }^{174}$

168 Complément à préposition, à la place de l'ablatif sans préposition.

169 loci - génitif à la place du datif (voir également Getica, 50:" loci nomen dedit....).

170 Spaniam pour Hispaniam. Chez Jordanès on ne retrouve que les formes Spania (pluriel: Spaniae) et Spani, et celle-ci, très souvent même.

171 rapta - le sujet, sous-entendu, est tempestas, exprimé dans la subordonnée temporelle.

172 perfraegit, pour perfregit - erreur des copistes, ou hyperurbanisme de Jordanès?

173 D'autres éditions de Florus présentent, à la place de la locution quam mox, cum uix - ce qui complique d'une manière erronée le texte (voir l'édition de C. Halm, Leipzig, Teubner, 1854).

174 Thesaurus linguae Latinae (op. cit., 1963, VIII - 10, p. 1553, 1. 62-63) n'indique comme attestation de 
Le remplacement de quam par cum, sans entraîner des modifications dans le sens de la phrase, prouve qu'à cette époque ce n'était plus la conjonction en soi qui rendait compte du sens de la locution, mais l'adverbe mox.

En échange, Jordanès maintient l'adverbe statim de Florus - corrélatif de la locution.

A propos de cum marquant l'antériorité immédiate, quoique construit avec le plus-que-parfait du subjonctif. voir le commentaire infra.

$\mathrm{Si}$, initialement, il servait pour corrélatif aux conjonctions temporelles et, plus tard, il formait avec celles-ci des locutions conjonctives, l'adverbe mox devient finalement lui-même conjonction temporelle, avec le sens de "dès que". 175

Mox, mot vulgaire, inexistant chez César et rarement utilisé par Cicéron, apparaît souvent chez Tite-Live et Tacite. ${ }^{176}$ Jordanès l'emploie fréquemment et dans toutes les hypostases possibles, à savoir:

a) comme adverbe, avec le sens d'"immédiatement", "tout de suite", sans qu'il fonctionne comme corrélatif pour une conjonction temporelle, voir Romana, 178, 233, 296, 324, 360, 366 etc.; Getica, 53, 92, 141, etc. etc. etc. Chez Jordanès, mox apparait presque à chaque page, parfois même plusieurs fois dans la même page;

b) comme corrélatif pour $u b i$ et $u t$, de même que pour cum "uere temporale" et cum narratif indiquant l'antériorité immédiate;

c) dans des locutions conjonctives avec ubi et avec cum (voir les exemples ci-dessus);

d) fréquemment, en tant que conjonction marquant l'antériorité immédiate.

Attesté d'abord chez Florus (première moitié du IIe siècle de notre ère) avec la valeur d'une conjonction, mox -conjonction devient de plus en plus fréquent en latin tardif (il apparaît, par exemple, en tant que conjonction chez Commodien. Instr., 1, 24, 19; Juvencus, Lucifer de Cagliari, Apicius, Ammien emploient la conjonction mox; on la retrouve dans la Peregrinatio Aetheriae, dans les inscriptions - voir C. E., 245, 2 etc. etc. Grégoire de Tours utilise la locution mox ut, mais jamais la conjonction mox ${ }^{177}$; Orose, Cassien et d'autres auteurs imitant le style classique ignorent la conjonction mox).

la locution cum mox que Célius Aurélianus, Chron., 4, 2, 16 et Jordanès (l'exemple cité plus haut). Mais elle apparaissait, probablement, chez d'autres auteurs tardifs aussi.

Pour ce qui est de la locution mox cum, celle-ci est ancienne (depuis Plaute) et elle réapparaît aux époques classique et impériale (Virgile, Tite-Live, Velléius Paterculus, Columelle, Juvénal, Suétone et d'autres l'emploient). Elle est utilisée à l'époque tardive aussi, voir Vitae Patrum, etc.

Vbi mox est rare aussi, par rapport à mox ubi (voir J. B. Hofmann - A. Szantyr, II - 2, op. cit., p. $652^{1}$; voir aussi Thesaurus linguae Latinae, VIII - 10, op. cit., p. 1552, 1. 40 sqq.; ibid., 1. 53 sqq.).

$V t$ mox est lui aussi peu employé, en comparaison de mox ut (voir Thesaurus linguae Latinae, VIII - 10 , op. cit., p. 1552, 1. 30 sqq.; ibid., 1. 52 sqq.

175 Bon nombre d'adverbes et de locutions adverbiales ont une évolution similaire: statim, nunc, postea, ex eo, propter hoc etc. [voir Ein. Löfstedt, Vermischte Studien zur lateinischen Sprachkunde und Syntax, Lund-London-Paris, 1936, p. 47 sqq.; voir également R. Kühner - C. Stegmann, Ausfürliche Grammatik der lateinischen Sprache, II - 2, op. cit., p. 365, Anm. 2 (par. 208, 10, Anm. 2).]

176 Voir Fried. Stolz - J- G. Schmalz, op. cit., p. 759; J. B. Hofmann - A. Szantyr, op. cit., II - 2, p. 6372. 
La conjonction mox sert initialement à marquer l'antériorité d'une action momentanée par rapport à une autre action momentanée. Dans les siècles suivants, mox sert également à introduire une action momentanée (celle de la subordonnée), tandis que la principale rend une action momentanée (le plus souvent), ou durative. L'imparfait de l'indicatif est extrêmement rare dans la subordonnée - un seul exemple cité dans le Thesaurus linguae Latinae. ${ }^{178}$ L'imparfait du subjonctif est, lui-aussi, rarement attesté179. Le présent de l'indicatif apparait seulement depuis Commodien et Ammien et il reste d'usage rare, surtout par rapport à l'emploi du parfait et du futur antérieur de l'indicatif. ${ }^{180}$

La conjonction mox apparaît chez Jordanès quatorze fois - donc elle est plus fréquente que $u b i$ et $u t \mathrm{~d}$ ' antériorité. ${ }^{181}$ La conjonction mox dépasse même en fréquence le corrélatif mox, ou le mox des locutions conjonctives. Mais la conjonction mox est dépassée de loin, chez Jordanès, par mox-adverbe qui contribue, aux côtés de ilico et de certaines locutions, à remplacer statim $^{182}$, cet adverbe mox qui ne remplit pas le rôle de corrélatif d'une conjonction, moins encore d'une locution conjonctive temporelle - voir d'ailleurs le commentaire ci-dessus sur l'adverbe mox.

Voici maintenant la répartition de la conjonction mox dans les deux ouvrages; sept exemples dans les Romana, sept exemples dans les Getica. Il nous faut cependant dire que tant les exemples des Romana que ceux des Getica ne semblent avoir été empruntés à un autre auteur (on ne peut toutefois l'affirmer avec certitude, vu que les principales sources d'inspiration pour les Getica - à savoir Getica enodia de Dion Chrysostome et l'Histoire des Goths de Cassiodore - se sont perdues). Voici pourtant un exemple où la construction grammaticale appartient indubitablement à Jordanès: "Sed cum subiectio omnium Maurorum facta est ${ }^{183}$, Iubas rex ... mox superatum se sensit, ueneno hausto defecit..." (Rom., 211), par comparaison à Rufus, 4: "Sed subacta omni Africa Mauros Iuba res tenebat, qui ... mortem sibi propria uoluntate consciuit." La parité entre les exemples de subordonnées introduites par mox dans les Roma$n a$ et ceux qui se trouvent dans les Getica prouverait, de plus, que les constructions avec mox appartiennent en propre à notre auteur.

177 Voir M. Bonnet, Le latin de Grégoire de Tours, op. cit., p. 320.

178 Voir Thesaurus linguae Latinae, op. cit., p. 1553, 1. 40 sq.

179 Voir Thesaurus linguae Latinae, op. cit., p. 1553, 1. 42-43.

180 Voir Thesaurus linguae Latinae, op. cit., p. 1553, 1.62 sqq.

181 Th. Mommsen, dans son Index de problèmes grammaticaux, n'enregistre pas tous les exemples de mox-conjonction voir op. cit., p. 193).

182 Relativement au remplacement de statim par d'autres adverbes, voir aussi notre commentaire dans les pages suivantes.

183 Voir, p. 49 de la $I^{\grave{r} r e}$ partie de cette étude, la discussion de cette proposition introduite par cum. Pour l'alternance de cum narratif avec des conjonctions "simplement temporelles" voir A. Ernout-Fr. Thomas, op. cit., p. 365, par. 361. 
Mox introduit toujours, chez Jordanès, des verbes exprimant des actions momentanées. En général, les subordonnées sont construites avec le parfait de l'indicatif. (d'après le modèle de $u b i$ et $u t$ ) - il y a onze exemples de ce type. Les trois autres exemples ont le verbe au subjonctif plus-que-parfait.

Dans la principale, c'est toujours l'indicatif parfait qui apparaît, bien que le présent fût plus convenable (pour ce qui est de l'emploi du présent historique dans les principales régissant des subordonnées temporelles d'antériorité en latin classique). Voici un exemple tiré des Romana: "... mox ei successit, ilico nulla faciente necessitate. .. Mesopotamiam Assyriamque et Armeniam Persis reliquid ${ }^{184}$...", Rom., 270; dans ce cas, la présence de ilico - en plus du modèle classique - justifierait l'emploi, dans la principale, du présent historique. Voir également Romana, 282: "Hic (Gordianus) etenim $^{185}$ mox Romae ${ }^{186}$ ingressus est, ilico Puppienum et Albinum, qui Maximino ${ }^{187}$ occidentes tyrannidem arripuissent, occidit, ... Parthis intulit bellum...".

Dans une seule phrase, la proposition principale de la temporelle introduite par mox a le verbe à l'indicatif présent - présent historique: Romana, 347 (dans la principale apparaît le corrélatif ilico).

Quant aux principales qui régissent des temporelles construites avec le subjonctif (voir ci-dessous), elles ont, toutes, le verbe au parfait de l'indicatif.

Un fait étrange est l'apparition du plus-que-parfait du subjonctif dans les subordonnées introduites par mox. Initialement ont été construites avec le subjonctif les locutions du type mox ubi, mox ut etc., plus tard les locutions telles ubi mox, cum mox etc.

Mox suivi du subjonctif plus-que-parfait est très rarement attesté avant Jordanès. Thesaurus linguae Latinae n'en enregistre que deux exemples antérieurs à Jordanès ${ }^{188}$ (Itala, Exod., 33, 9 et la traduction d'Oribasius, Synops., 9, 24, 23 Aa). ${ }^{189}$ Mox suivi du subjonctif plus-que-parfait réapparaît ensuite chez un auteur postérieur à Jordanès - Grégoire le Grand (pape de 590 à 604). L'imparfait du subjonctif après mox est encore plus rarement attesté (un seul exemple dans le Thesaurus linguae Latinae: Act. Archel. 31, $8^{190}$; aucun exemple chez Jordanès).

Dans les oeuvres de Jordanès, mox suivi du subjonctif plus-que-parfait apparaît trois fois: deux fois dans les Romana et une fois dans les Getica. Tous ces exemples

184 Pour ce qui est de reliquid, voir note 43.

185 etenim a, dans ce passage, la valeur de la particule ?? marquant le passage d'une phrase à l'autre.

186 ingredior avec le sens de "entrer", accompagné d'un complément au datif, apparaît aussi dans les Getica, 311 ("Romae ingreditur"). Voir cependant Romana, 163: "fretum ingressus est"; c'est toujours l'accusatif qui accompagne ingredior dans les Romana, 231; ibid., 373; Getica, 310 etc.

187 désinence d'ablatif à la place de la désinence d'accusatif, ou bien forme d'accusatif sans la finale - $m$ ? Voir, dans la même phrase, le datif classique: "Parthis intulit bellum".

188 voir vol. VIII - 10, op. cit., p. 1553, l. 26-33.

189 Dans la plus importante Grammaire de la langue latine: M. Leumann - J. B. Hofmann - A. Szantyr, II, 2, op. cit., cette construction de la conjonction mox n'est pas mentionnée.

190 Voir Thesaurus linguae Latinae, VIII - 10, op. cit., p. 1553, I. 42-43. 
diffèrent des modèles que Jordanès a suivis. En voici un, Romana, 372: "Qui Campa* nia $^{191}$ ingressus mox ad campos uenisset Barbaricos, ilico exercitus fauore ${ }^{192} .$. excepit." Cfr. Continuator Marcellini. ad an. 536: "qui mox in campo Barbarico regnum peruasit, expeditione soluta Romam ingreditur..." On peut observer que chez le Continuateur de Mercellinus ilico n'existe pas. Voir aussi Romana, 340, en comparaison de Marcellinus, 474. Voir également Getica, 60, par rapport à la traduction de Dictys, 4, 17-18.

La différence entre les modèles et la construction de Jordanès, le nombre assez grand d'exemples avec le subjonctif (par rapport aux exemples avec l'indicatif chez Jordanès), la proportion de dérogations plus grande dans les Romana que dans les Getica, l'apparition de cette construction chez des auteurs postérieurs à Jordanès sont autant d'arguments qui nous amènent à considérer qu'il ne s'agit pas de phénomènes fortuits ou de fautes ou innovations propres à l'auteur en question. Ce type de construction était, vraisemblablement, fréquent en latin vulgaire et ces attestations supplémentaires (de Jordanès) ne font qu'accrôttre l'importance des écrits de Jordanès.

Dans ces phrases, mox a le sens: "bientôt après que" et montre que l'action de la temporelle précède de peu l'action de la principale. L'antériorité immediate est encore plus clairement marquée si, dans la principale, on retrouve l'adverbe ilico (voir, cidessus, l'exemple des Romana, 372). Autrement, c'est le contexte et le sens de l'adverbe- conjonction mox qui indiquent l'antériorité récente. Quoiqu'il en soit, les subordonnées introduites par mox ne s'inscrivent pas dans la sphère des temporelles introduites par postquam, construit avec le parfait de l'indicatif.

L'unique corrélatif de la conjonction mox, chez Jordanès, c'est l'adverbe ilico. Celui-ci apparait souvent - il y en a six exemples. Ilico est un mot de prédilection des auteurs tardifs qui l'emploient même dans les locutions conjonctives ${ }^{193}$. Chez Jordanès, ilico apparaît souvent, soit comme adverbe de lieu, soit comme adverbe de temps remplaçant statim (ce dernier, présent, quand-même, chez Jordanès), ou bien en tant que corrélatif de diverses conjonctions et locutions (dum, ut primum).

Fait intéressant, le corrélatif ilico apparaît surtout dans les Romana et voici comment se présente la situation: pour cinq corrélatifs ilico de mox dans les Romana, il n'y a qu'un seul exemple dans les Getica. La présence répétée du corrélatif s'expliquerait par la tendance de l'auteur à construire avec plus de précision (en tout cas, une excessive précision) certains passages de Romana (voir Romana, 372 - exemple cité; voir aussi Romana, 296: "Hic (Diocletianus) ... mox in regno levatus est, ilico Aprum ... percussit.")

Ilico apparaît une seule fois comme corrélatif de mox construit avec le subjonctif (voir Romana, 372). Dans les autres cas, il se trouve dans des principales qui régissent mox construit avec l'indicatif parfait (Romana, 270, 282, 296, 347; Getica, 126).

191 forme d'accusatif où la finale - $m$ n'est pas marquée.

192 voir la note précédente.

193 voir J.B. Hofmann - A. Szantyr, Ul - 2, op. cit., p. 6371. 
Une autre conjonction qu'utilise Jordanès pour marquer l'antériorité immédiate, c'est quod.

Dans le latin vulgaire de l'époque tardive, quod devient conjonction universelle ${ }^{194}$, annonçant, de la sorte, les valeurs multiples de la conjonction "ca" en roumain, "che" en italien (et rhéto-roman), "que" en français, espagnol, portugais. ${ }^{195}$

Dans les écrits de Jordanès, quod peut assumer un sens comparatif (dans une subordonnée comme "quod solet", à la place de "ut solet"), un sens final, un sens temporel, en plus de ses valeurs usuelles, introduisant des propositions relatives, des propositions-sujets, des complétives et des causales.

Cependant, quod temporel est très rare chez Jordanès: deux exemples seulement. Voir Romana, 203: "Sed hunc quoque tantum quod ab Alpe ${ }^{196}$ descenderat apudque 197 Metaurum castra metantem Claudius Nero cum Liuio Salinatore debellat." Cfr. Florus, 1, 22, 50: "Set hunc quoque, tantum quod ab Alpe descenderat, aput Metaurum castra metantem Claudius Nero cum Liuio Salinatore debellat." Voici la traduction de cette phrase: "Mais Claudius Néron et Liuius Salinator vainquent même celui-ci (Hasdrubal), dès qu'il fut descendu des Alpes, pendant qu'il délimitait son camp, près de Métaure".

On remarque qu'il s'agit d'une locution tantum quod, formée de deux mots très utilisés en latin vulgaire (chez Jordanès, tantum est fréquent, en corrélation ou non avec des conjonctions de subordination; parmi celles-ci, il faut citer les comparatives et, notamment, les consécutives ${ }^{198}$ ). Le sens temporel de cette locution est issu de sa valeur comparative, plus ancienne (voir également l'apparition des locutions in tantum quia, in tantum quod en latin médiéval, à valeur comparative, temporelle - de simultanéité, d'antériorité et même de postériorité, en d'autres cas -à nuance causale ou conditionnelle ${ }^{199}$ ).

Dans un autre exemple, quod temporel a une nuance adversative-concessive ("quand" - "bien que"): “... et quod nobis uidetur sol ab imo surgere, illos ${ }^{200}$ per terrae marginem dicitur circuire 201 ", Get., 20.

194 Voir J. B. Hofmann - A. Szantyr, II - 2, op. cit., par. 313, pp. 579-582; D. Norberg, Syntaktische Forschungen auf dem Gebiete des Spätlateins, Uppsala, 1943, p. 232 sqq.; J. B. Hofmann, Lateinische Umgangsprache, Heidelberg, 1957, 3. Aufl., p. 722 sqq.

195 Voir F. Brunot, Histoire de la langue française, Paris, 1924, vol. I, pp. 216-17; C. Tagliavini, Origenes de las lenguas neolatinas, Mexico, 1973, p. 323; etc.

$196 a b$ Alpe - singulier poétique au lieu du pluriel (même forme chez Florus).

197 -que superflu. D' ailleurs, dans le fragment correspondant de Florus cette particule n'existe pas (voir le texte cité ci-dessus). Pour -que superflu chez Jordanès, voir aussi Th. Mommsen, Index grammatical à l'édition mentionnée, p. 194.

198 Voir aussi la corrélation: "in tantum - ut" (à valeur consécutive) chez Jordanès (Getica, 62 et 255).

199 Voir, à ce propos, R. Iordache, "In quantum", "in tantum", locuciones del latín imperial, dans "Helmantica", N 99, Salamanque, 1981, pp. 327-28 et pp. 332-333.

200 illos, pour illis. 
B) Pour indiquer une antériorité accentuée; l'écrivain utilise les conjonctions cum narratif, postquam et dum (énumérées par ordre de leur fréquence chez Jordanès).

Cum est en général construit avec le plus-que-parfait du subjonctif, tandis que le verbe de la principale est: a) d'habitude, à l'indicatif parfait; b) parfois, à l'imparfait de l'indicatif; c) rarement, au présent historique; d) une seule fois, au plus-que-parfait de l'indicatif (employé pour raconter plus rapidement les événements).

Dans une seule phrase, cum est suivi du parfait du subjonctif, voir Getica, 257: "Cum que haec omnia prouentu felicitatis egerit, non uulnere hostium, non fraude suorum, sed gente incolume ${ }^{202}$ inter gaudia laetus sine sensu doloris occubuit." (phrase concernant la mort d'Attila). On remarque, ici, la perte de la valeur primitive, explicative, de cum narratif. Dans d'autres exemples aussi (où il apparaît même avec le plus-que-parfait du subjonctif), cum est dépourvu de sa valeur originelle, instrumentale-modale. Voir Getica, 307: "Eodem namque tempore ... cum per fidelissimum suum patricium Belesarium ${ }^{203}$ reportasset triumphum, nec mora in ipso tempore ${ }^{204} \ldots$ per eundem ducem mouit procinctum." (remarquer les corrélatifs" "eodem tempore", "nec mora", "in ipso tempore").

Cum historique, construit avec le plus-que-parfait du subjonctif apparaît parfois chez Jordanès pour exprimer une antériorité immédiate plutôt qu'une antériorité accentuée. Le degré d'antériorité est indiqué par le contexte et les adverbes corrélatifs. Voir Romana, 51: "Quos uagientes meretrix quaedam Lupa nomine cum audisset, statim tollens ad Faustulum pastorem adduxit."; voir également Getica, 141: "Quod cum Gratianus imperator ... conperisset, mox ad eos collecto uenit exercitu...". Voir aussi l'exemple déjà cité - Romana, 307.

L'adverbe iam apparaît lui aussi en tant que corrélatif de cum narratif d'antériorité, voir Romana, 170: “... cum terrorem nominis sui latae ${ }^{205}$ circumtulisset, cumque magnam uim iuuentutis ducesque ipsos aut cepisset aut haberet in uinculis classemque ingenti praeda onustam ... praemisisset, iam ipsam belli caput Chartaginem ${ }^{206}$ urguebat..." (passage copié sur Florus, 2, 2, 21).

L'adverbe tum peut servir aussi comme corrélatif pour cum narratif construit avec le plus-que-parfait du subjonctif, voir Romana, 242 (passage copié, en général, sur Florus, 4, 12, 7).

201 forme courante en latin vulgaire, au lieu de circumire.

202 incolume pour incolumi. Le même adjectif à l'ablatif apparaît avec la voyelle finale $e$ au lieu de $i$, dans les Getica, 68. A propos de la confusion entre $e$ et $i$, voir les notes $46,64,67 \mathrm{etc}$.

203 Chez Jordanès on retrouve pour Belisarius soit la forme Belesarius, soit Belezarius (celle-ci, une seule fois). Pour Belisarius, voir Continuator Marcellini, an. 545.

204 Complément à préposition, è la place du complément sans préposition.

205 latae pour late, hyperurbanisme.

206 Chartaginem à la place de Carthaginem (même faute dans Romana 168 etc.). La forme erronée apparaît presque partout chez Jordanès. 
Le corrélatif tandem apparaît, dans un exemple (Romana, 104), pour cum narratif indiquant l'antériorité non déterminée.

En tout cas, cum construit avec le subjonctif plus-que-parfait (indiquant d'habitude l'antériorité non déterminée et, plus rarement, une antériorité immédiate) est mieux conservé dans les ecrits de Jordanès que cum narratif de simultanéité (voir aussi notre commentaire ci-dessous sur postquam et dum d'antériorité).

Cum suivi du plus-que-parfait du subjonctif est rare dans les Getica par rapport à sa fréquence dans les Romana (cfr. ci-dessous la fréquence de l'emploi de postquam et dum construit avec le plus-que-parfait du subjonctif dans les Getica).

Le sens "après que" est assez souvent rendu, chez Jordanès, par la conjonction postquam (seize exemples dont trois à nuance causale; une autre fois, postquam a le sens de "depuis que").

Fréquemment employé en latin vulgaire dès l'époque préclassique, postquam l'emporte même sur cum narratif, en bas latin.

La répartition de la conjonction postquam dans les deux ouvrages se fait de manière égale: huit exemples dans les Romana (dont les trois à nuance causale) et huit dans les Getica.

Si pourtant, chez Jordanès, la fréquence de postquam ne dépasse pas celle de cum, ce qui étonne, c'est la manière dont il est construit, de même que l'emploi fréquent du subjonctif. Ainsi, postquam est, dans huit exemples suivi de l'indicatif (huit conjonctions postquam pour dix verbes) et, dans huit autres phrases, donc une proportion de $50 \%$, du subjonctif plus-que-parfait (huit conjonctions suivies de onze verbes).

Voici la distribution de l'emploi du subjonctif pour chaque ouvrage: dans les Romana, postquam est, par quatre fois, suivi du subjonctif (quatre conjonctions quatre verbes); dans les quatre autres cas (quatre conjonctions - cinq verbes), c'est l'indicatif qui suit; dans les Getica nous retrouvons, en principe, la même situation: quatre exemples avec le subjonctif (quatre conjonction - huit verbes) et quatre, avec l'indicatif (quatre conjonctions - cinq verbes).

En comparant les phrases construites avec le subjonctif et les modèles dont Jordanès s'est inspiré et vu, d'autre part, la parité entre les exemples des Romana et ceux des Getica, on constate qu'il s'agit là de constructions bien propres à Jordanès. On s'aperçoit, de plus, que les verbes au subjonctif se succèdent naturellement, après la même conjonction, dans une seule phrase (voir Getica, 110 , où il y a quatre verbes au plus-que-parfait du subjonctif). Dans un autre exemple, postquam est suivi tant du subjonctif plus-que-parfait, que du subjonctif imparfait (Getica, 304).

On ne saurait expliquer l'emploi du subjonctif après postquam ni par le style direct, ni par l'attraction modale. Voir Romana, 265: “... Iohannem apostolum et euangelistam, postquam in feruente ${ }^{207}$ oleo $^{208}$ missum non potuisset extingui ${ }^{209}$, Patmo $^{210}$

207 feruente à la place de feruenti - confusion des voyelles e et $\mathrm{i}$, ou bien forme d'accusatif sans - $m$ final (voir 
eum insulam relegauit ubi apocalypsim uidit." Voici la traduction du fragment: "Après que - puisque - cela fut impossible, c'est-à-dire tuer l'apôtre et évangéliste Jean, même jeté dans de l'huile bouillante, Domitien le relégua dans l'île de Pathmos, où Jean vit l'Apocalypse."

Voir également Romana, 267: "Hic (Traianus) ... Hiberos ... et Sauromatas ... edomuit, postquam ad feritatem prorupissent." Voir aussi Romana, 53, 376, Getica, 110,123 et 304 . Ajoutons un exemple où postquam indique le commencement de l'action de la principale - Getica, 163.

L'emploi du subjonctif après postquam ou posteaquam (en général, il s'agit du plus-que-parfait et, moins souvent, de.l'imparfait) est un trait de latin vulgaire, attesté pour la première fois chez Cicéron (subjonctif plus-que-parfait en Man., 9; Dei., 36; Att., 11, 12, 1; Leg., 2, 64; subjonctif imparfait en Cluent., 181; Fam., 2, 19, 1 etc.); plus tard, le subjonctif apparaît dans Bellum Afr., 91, 3; 93, 3; Vitruve, 2, 9, 16; TiteLive, 22, 1, 2; Hyginus, Fab., 140, 5; Valère Maxime, 5, 7 ext. 2 et, de plus en plus souvent en latin tardif, chez Ammien, 30, 7, 11; dans Collectio Avellana, p. 26, 27; chez Augustin, Cassiodore etc. (contrairement à l'opinion de certains chercheurs ${ }^{211}$, selon lesquels il n'existerait, durant toute la latinité, que douze exemples environ de postquam et posteaquam suivis du subjonctif - tant plus-que-parfait, qu'imparfait).

L'emploi du subjonctif après postquam et posteaquam s'explique par l'influence de la construction de cum narratif - d'ailleurs, rien que chez Jordanès nous comptons huit conjonctions suivies de douze verbes au subjonctif (y compris à l'imparfait du subjonctif). Si cum était tombé en désuétude à l'époque tardive, le modèle de ses constructions était encore vivant et, comme nous l'avons vu, plusieurs conjonctions sont construites avec le subjonctif plus-que-parfait et imparfait.

Cependant, lorsqu'il s'agit d'expliquer certaines constructions avec le subjonctif, il faut parfois tenir compte aussi de la perte - en bas latin - de la valeur modale (volitive ou potentielle) du subjonctif, notamment du subjonctif plus-que-parfait.

On constate assez fréquemment, chez Jordanès, l'apparition - dans les subordonnées introduites par postquam - de formes du subjonctif plus-que-parfait, İ̀re conjugaison, sans le suffixe - $u$-, voir: regnassent (Rom., 53), fugasset, depraedasset, superasset (ces trois derniers exemples dans une seule phrase des Getica, 110; c'est toujours ici qu'apparaît le subjonctif adtriuisset). Mais, comme de pareilles formes apparaissent chez Jordanès dans des propositions introduites par quamuis également (voir

la note suivante).

208 in oleo - complément à l'ablatif, à la place de l'accusatif de direction, ou bien accusatif présentant la voyelle o à la place de u (phénomène fréquent dans les deux ouvrages) et sans la finale $-m$ ?

209 Pour l'interprétation du syntagme "non potuisset exstingue", voir R. Iordache, L'infinitif dans les oeuvres de Jordanès, op. cit., pp. 139-40. Posse apparaît ici comme unipersonnel, accompagné de la construction "Accusatiuus cum Infinitiuo".

210 Pathmo à la place de Pathmum. Cependant, un peu plus loin apparaît la graphie correcte insulam.

211 Par exemple R. Kühner - C. Stegmann, II - 2, op. cit., p. 358, 8. 
Romana, 214: conspirassent) et comme l'absence du suffixe peut être constatée à l'indicatif aussi, dans d'autres types de propositions (voir inportarunt, Rom., 321; trucidarunt, Get., 111, et 258; famularunt, Get., 115; inchoarunt, Get., 245; occuparat, Rom., 95 etc. etc.) et que, d'autre part, les passages en question diffèrent nettement des modèles dont Jordanès a repris les idées, nous considérons que dans les exemples ci-dessus (regnassent - Rom., 53 etc.) il ne s'agit pas de l'emploi du subjonctif plus-que-parfait à la place de l'indicatif plus-que-parfait.

Parmi les conjonctions qui subissent l'influence de cum narratif, postquam présente, à un moment donné, une situation spéciale, regroupant dans une même phrase tant le subjonctif plus-que-parfait que le subjonctif imparfait, voir Getica, 304: "Sed postquam ad senium peruenisset et se in breui ${ }^{212}$ ab hac luce egressurum cognusce$r e t^{213}$, conuocans Gothos comites gentisque suae primates Athalaricum infantulum...., filium filiae auae Amalasuenthae, ... regem constituit...". ("mais après qu'il eut vieilli (Théodéric) et qu'il comprit que bientôt il quitterait ce monde, convoquant les comtes goths et les citoyens les plus notables de son peuple, il établit roi le petit Athalaric, fils de sa fille Amalasuenthe.")

Profitons de cette occasion pour préciser que dans les écrits de Jordanès, seuls cum narratif et postquam rendent possible la réunion, dans la même phrase, du plusque-parfait et de l'imparfait (du subjonctif). Voir Romana, 170: "sed omnium uictor Regulus cum terrorem nominis sui latae ${ }^{214}$ circumtulisset, cumque magnam uim iuuentutis ducesque ipsos aut cepisset aut haberet in uinculis classemque ingenti praeda

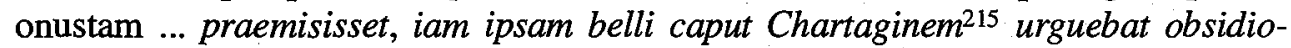
ne...".

L'emploi du subjonctif imparfait dans la deuxième subordonnée des Getica, 304 a pour résultat le glissement de cette proposition de la sphère de l'antériorité non déterminée vers la sphère de l'antériorité récente. Pour la deuxième subordonnée, postquam ne peut garder le sens de "après que", mais acquiert le sens: "dès que", ou "quand", "comme".

Le subjonctif imparfait précédé par postquam est assez rare en latin (voir les exemples extraits de Cicéron, supra, ensuite Tite-Live, 22, 1, 2; 4, 13, 10 - voir l'exemple ci-dessous). Chez Jordanès. ce temps du subjonctif n'est utilisé qu'une seule fois (voir aussi, dans les pages suivantes, notre commentaire sur la faible fréquence de l'imparfait de l'indicatif chez Jordanès). Dans cet unique exemple de Jordanès, l'imparfait du subjonctif n'est pas indépendant, mais il semble réclamé par le plus-que-parfait (du même mode) qui accompagne postquam; en réalité, cette construction avec le plus-que-parfait domine la phrase tant par sa position (par l'ordre de

212 in breui - expression vulgaire pour breui (voir également Getica, $233 \mathrm{etc}$.).

213 cognusceret pour cognosceret. Pour la confusion des voyelles $o$ et $u$, voir les notes antérieures.

214 au lieu de late.

215 pour Carthaginem. 
succession des subordonnées), que par son importance sémantique dans le passage en question (Cf. Tite-Live, 4, 13, 10: "Quae postquam sunt audita et undique primores patrum et prioris anni consules increparent, ... tum Quinctius consules inmerito increpari ait..."). Bien que le deuxième subjonctif soit conditionné par le premier, l'apparition des deux types de subjonctif après postquam est toutefois une tentative intéressante à attribuer les deux valeurs d'une conjonction vieillie, cum, à postquam, cette dernière répandue en latin vulgaire.

Pour ce qui est de l'emploi des temps de l'indicatif, on ne constate généralement pas de dérogations aux normes classiques. Statistiquement, la situation se présente ainsi: six conjonctions sont suivies de huit verbes au parfait de l'indicatif, une conjonction, par un verbe au plus-que-parfait et une autre, par un verbe à l'imparfait.

Quant à l'emploi du plus-que-parfait, voir Romana, 317: "Hic etenim Eugenius confisus uiribus Arbogasti postquam apud Viennam Valentinianum extincxerat ${ }^{216}$, regnum inuasit...".

En latin cultivé classique, l'indicatif parfait aurait été, dans ce cas, préféré. Les subordonnées contenant le plus-que-parfait (de l'indicatif) sont assez fréquentes chez Tite-Live et Tacite ${ }^{217}$. A l'époque tardive, le plus-que-parfait n' accompagne pas souvent postquam - d'ailleurs, il n'y en a qu'un seul exemple chez Jordanès. Notons aussi que l'exemple de Jordanès n'est dû à l'influence d'aucun autre écrivain (cf. Orose, 7, 35 , où Jordanès puise son idée).

L'imparfait de l'indicatif, rarement utilisé à l'époque classique (et plus rarement encore en latin préclassique), est employé dans une seule subordonnée - Romana, 135: "mox eosdem, postquam esse homines liquebat, alioquin nihil respondere dignantes pari uecordia mactant facesque tectis iniciunt et totam urbem igni ferro manibus exaequant." "'Aussitôt qu'ils se sont convaincus - puisqu'il était clair - que ceuxlà étaient des gens qui d'ailleurs ne daignaient nullement leur répondre, peu de temps après ils les sacrifient avec une égale fureur et lancent des torches sur les toits et détruisent la ville entière par le feu, par le fer et de leurs mains mêmes."). Le passage est, du reste, copié sur Florus, 1, 13, 14 .

La nuance causale de cette subordonnée est évidente. L'imparfait employé dans cet exemple confere plus d'élégance à l'expression.

Les règles de la Concordance des temps sont respectées. Dans les principales régissant les subordonnées qui commencent par postquam on utilise d'habitude le parfait de l'indicatif, parfois le présent historique (voir les exemples ci-dessus, Roma$n a, 135$ et 380), une seule fois, le participe présent avec la valeur d' un indicatif imparfait (voir Getica, 123: "Quorum natio saeua ... uenationi ${ }^{218}$ tantum ${ }^{219}$ nec alio labore

216 extincxerat, pour exstinxerat (voir aussi Romana, 385: extincxit, Romana, 203: iuncxisset etc.).

217 Voir R. Kühner - C. Stegmann, II -2, op. cit., pp. $355^{4}-356$, par. $207,4$.

218 uenationi-forme d'ablatif (voir plus loin: "alio labore"). La confusion des voyelles $e$ et $i$ en position finale se retrouve fréquemment dans les pages des ouvrages de Jordanès. Voir aussi note 46. 
experta, nisi quod, postquam creuisset in populis ${ }^{220}$, fraudibus et rapinis uicinarum gentium quiete 221 conturbans").

Une autre conjonction qu'utilise Jordanès pour rendre autant l'antériorité non déterminée, que l'antériorité immédiate, c'est dum construit avec le plus-que-parfait du subjonctif.

Dum suivi du subjonctif plus-que-parfait apparaît dans les Romana à quatre fois; en revanche, il apparaît à sept fois dans les Getica. Quant à la distribution de cum narratif suivi du subjonctif plus-que-parfait, celle-ci est inversement réalisée dans les deux ouvrages de Jordanès: cum narratif d'antériorité est de loin plus fréquent dans les Romana que dans les Getica.

Ce type de dum a souvent une valeur explicative-qualificative-temporelle, tout comme cum narratif. Voir Getica, 249: “...dum utrique ad se uenissent, Balamber sagitta missa caput Venetharii saucians interemit...”. Dans certains exemples, le caractère explicatif-causal est évident, voir Romana, 330: “... Bonifatius, dum in offensa ${ }^{222}$ Valentiniani uenissset, malo publico se defendere uoluit...". Pour la valeur sémantique complexe de ces subordonnées plaide aussi l'antéposition des propositions introduites par dum par rapport aux principales. Voir également la corrélation "dum -sic" (Getica, 307).

Les propositions introduites par dum et ayant le verbe au subjonctif plus-que-parfait indiquent, en général, une antériorité non déterminée. Parfois c'est le contexte et les corrélatifs qui confèrent à la subordonnée temporelle la qualité de présenter l'antériorité immédiate. Voici un exemple tiré des Getica, 307: "quod dum Iustinianus imperator Orientalis audisset..., sic est commotus." (Cf., pour cet exemple, Continuator Marcellini ad an. 534: "Cuius mortem imp. Iustinianus ut doluit, sic et ultus est.").

Ce qu'il y a d'important c'est que la manière de construire ces subordonnées n'est pas copiée sur quelque historien antérieur ou contemporain (naturellement, sous la réserve de ne pas avoir eu la possibilité de consulter l'ouvrage sur les Gètes de Dion Chrysostome et 1'Histoire des Goths de Cassiodore, tous les deux perdus depuis longtemps).

Comme auxiliaire des verbes, on rencontre parfois "fuisset" à la place de esset phénomène qui, chez Jordanès, se retrouve dans d'autres types de propositions aussi (voir Romana, 293: “dum ... missus fuisset”; ibid., 355: “... dum peremptus fuisset..."); dans un seul cas on rencontre un verbe de la I ${ }^{\text {ère }}$ conjugaison sans le suffixe $-u$ (Getica., 248: "imperasset") - voir, au sujet de cette forme de plus-que-parfait (sans le suffixe $-u$-), notre commentaire supra. Les subjonctifs utilisés dans les subordonnées

219 L'adverbe tantum au sens de "seulement" est fréquent chez Jordanès (voir, au sujet de tantum, la discussion ci-dessus).

220 in populis - à la place de l'ablatif non prépositionnel, ou de l'ablatif précédé par ex.

221 accusatif singulier, avec omission de la consonne finale.

222 accusatif,; omission du $-m$ final. 
introduites par dum sont: uenisset, au singulier et au pluriel aussi, uidisset (voir les exemples précités et, en plus, Romana, 320 et Getica, 122); apparaissent aussi, à une seule fois chacun: ualuisset (Getica, 47), praecipisset ${ }^{223}$ (Getica, 129), mouisset (Getica, 236), audisset (Getica, 307) - verbes qui sont habituels dans les subordonnées introduites par cum narratif.

Dum accompagné du plus-que-parfait du subjonctif, soit avec la valeur de cum narratif d'antériorité, soit avec celle de postquam simplement temporel, est rarement attesté avant Jordanès (le premier exemple apparaît, selon toutes probabilités, chez Hygin, Fab., 18, ensuite dans la traduction de l'oeuvre de Hermas, Vulg. uis., 1, 1, Fulgence de Ruspe, Praedest., 3, $22^{224}$, parfois chez Cassiodore - importante personnalité culturelle du VIe siècle; on pourrait parfois supposer une attraction modale, voir Claudius Tryphoninus, Digesta, 49, 15, 12, 9 et Pseudo Augustinus, Quaest. test., 1, 73 , p. $125,26^{225}$ ).

L'absence de cette construction de dum chez les écrivains antérieurs à Jordanès peut s'expliquer, en quelque mesure, par le fait que l'emploi du plus-que-parfait avait pour résultat l'apparition d'une valeur nouvelle (d'antériorité non déterminée) pour $d u m$, valeur qui ne s'accordait pas très bien avec les sens fondamentaux de cette conjonction. D'ailleurs, chez Jordanès, le remplacement de cum narratif de simultanéité par dum est de beaucoup plus fréquent que l'emploi de dum à la place de cum narratif d'antériorité. C'est pour cela que cum narratif de simultanéité apparaît si rarement dans les écrits de Jordanès; d'autre part, on constate que dum d'antériorité est moins utilisé que cum narratif d'antériorité.

Dans les oeuvres de Jordanès on rencontre donc onze exemples de dum suivi du subjonctif plus-que-parfait, par conséquent un nombre de constructions de dum supérieur à celui des postquam (postquam suivi du plus-que-parfait du subjonctif) et, de toute façon, un nombre d'exemples assez important par rapport à tous ceux qu'indique le Thesaurus linguae Latinae pour dum temporel et même pour dum à nuance concessive et $d u m$ à nuance causale 226

Cette habitude de construire $d u m$ avec le subjonctif plus-que-parfait se laisse voir aussi dans le fait que, parfois, une seule conjonction est suivie de deux verbes, les deux étant au subjonctif plus-que-parfait. Voir Getica, 122: "Quas spiritus inmundi

223 praecipisset, au lieu de praecepisset.

224 les derniers exemples sont indiqués dans le Thesaurus linguae Latiane, V - 1, op. cit., p. 2220, lignes 58-59.

225 Voir Thesaurus linguae Latinae, V - 1, op. cit., p. 2220, lignes 60-62.

226 Voir Thesaurus linguae Latinae, V - 1, op. cit., pp. 2220, 2221, 2222. Pour ce qui est de la rareté de cette construction, nous renvoyons également à l'opinion, exagérée d'ailleurs, de H. Goelzer: "Cassiodore va même jusqu'à employer après dum le plus-que-parfait et le parfait du subjonctif, construction extrêmement rare avant lui, pour ne pas dire tout à fait inconnue." (Etude lexicographique et grammaticale de la latinité de Saint Jérôme, op. cit., p. 359). C'est nous qui soulignons. 
per herimum ${ }^{227}$ uagantes dum uidissent et eorum conplexibus ${ }^{228}$ in coitu miscuissent, genus hoc ferocissimum ediderunt, quae ${ }^{229}$ fuit primum inter paludes...".

Dans d'autres passages, dum accompagné du plus-que-parfait du subjonctif alterne tout naturellement avec cum narratif d'antériorité (voir Getica, 307).

La présence répétée, chez Jordanès, de dum suivi de subjonctif plus-que-parfait peut constituer un indice de l'emploi plus fréquent - de cette manière - de dum en latin vulgaire au VIe siècle de notre ère (voir également, dans ce sens, les six exemples de dum construit avec le subjonctif plus-que-parfait qui apparaissent chez Eugippius, écrivain du commencement du $\mathrm{VI}^{\mathrm{e}}$ siècle $^{230}$; voir également la note 226 concernant l'existence de cet usage chez Cassiodore).

Dans un seul cas, dum est construit avec le parfait du subjonctif, voir Getica, 214: "Verum inter has obsidionum moras Vesegothae regem, fili ${ }^{231}$ patrem requirunt, admirantes eius absentiam, dum felicitas fuerit subsecuta ${ }^{232}$." Le mode s' expliquerait, dans cet exemple, par l'idée de style indirect. Quoi qu'il en soit, c'est l'unique exemple du subjonctif parfait utilisé dans divers types de subordonnées introduites par dum. D'autre part, nous constatons que le subjonctif parfait est extrêmement rare dans les propositions introduites par cum (un seul exemple dans la subordonnée temporelle voir notre commentaire supra; un exemple dans une subordonnée explicative-causale et un autre dans une subordonnée concessive).

Nous assistons parfois à une manière impropre d'utiliser dum - avec l'imparfait du subjonctif, pour exprimer une relation temporelle d'antériorité. Voir Romana, 287: "Valerianus et Gallienus, dum unus ${ }^{233}$ in Retia ${ }^{234}$ a militibus, alter Romae a senatu in imperio $^{235}$ leuarentur ${ }^{236}$, regnauerunt an. XV".

Dans une autre phrase, dum est suivi tant de l'imparfait du subjonctif que du parfait de l'indicatif: "Secundo dum Lucius Lucullus a Pontico regno Tigranem Armeniae regem ... expelleret, omnemque Armeniam inuasam ${ }^{237}$ ad Mesopotamiam uenit,

227 herimum, à la place de eremum (voir aussi heremo pour eremo-Romana, 28).

228 conplexibus, pour complexibus. Pour ce phénomène, voir les notes antérieures.

229 quae pour quod.

230 Voir index uerborum et locutionum pour l'édition de Eugippius établie par P. Knoell, Vienne, 1885 (p. 89), en “C. S. E. L.", vol. IX.

231 fili, à la place de filii (voir également Getica, 259).

232 On remarque l'emploi de l' auxiliaire fuerit au lieu de sit. Cf.: "Si uictus fuero, uestra pietas nihil amittit.", Get., 291.

233 unus est employé ici pour alter (un autre exemple de ce genre apparaît dans les Romana, 272; plus nombreux sont les exemples où unus est utilisé à la place de alius).

234 pour Rhaetia.

235 au lieu de in imperium.

236 pluriel, à la place du singulier.

237 omnem Armeniam inuasam - accusatif absolu. 
ibi Nitziben cum fratre regis Parthorum cepit, aequa sorte Persidam ${ }^{238}$ cupiens deuastare..." (Rom., 232).

L'apparition de l'imparfait du subjonctif à la place du plus-que-parfait du subjonctif est due à un style négligent, mais elle peut être aussi un hyperurbanisme, une réaction contre un phénomène beaucoup plus fréquent en bas latin - l'emploi du subjonctif plus-que-parfait au lieu de l'imparfait du même mode (voir note 129).

Chez Jordanès, le plus-que-parfait du subjonctif apparaît cependant rarement à la place de l'imparfait du subjonctif (voir notre commentaire page 58 de la première partie de cette étude). Quant à l'utilisation de l'imparfait à la place du plus-que-parfait du subjonctif, elle est évidente dans certains passages de Jordanès, par exemple Getica, 210 (“... pene ${ }^{239}$ Attilam trucidarent ${ }^{240}$, nisi ${ }^{241}$ prouidus prius fugisset et se suosque ilico intra septa ${ }^{242}$ castrorum ... reclusisset'). Cependant, l'emploi de l'imparfait à la place du plus-que-parfait du subjonctif est rare chez Jordanès. D'ailleurs, ce phénomène n'est que rarement attesté à l'époque tardive. ${ }^{243}$

Les règles de la Concordance des temps sont en général correctement appliquées. D'ordinaire, le verbe de la principale est à l'indicatif parfait; dans deux cas, il est au présent historique (Romana, 330 et Getica, 236). Dans un seul cas, il se trouve, clans la principale, un participe présent (qui dépend d'un présent historique de la même phrase) - il s'agit de la principale qui régit un dum construit avec le subjonctif parfait (l'exemple déjà présenté - Getica, 214).

Pour les conjonctions d'antériorité non déterminée proprement dite et, parfois, d'antériorité immédiate, les corrélatifs sont extrêmement rares. Ainsi cum indiquant l'antériorité non déterminée a, dans un cas, pour corrélatif tandem (voir notre commentaire supra).

Cum suivi du subjonctif plus-que-parfait a, d'autres fois, pour corrélatifs mox, statim, iam et tum, dont le rôle est de rapprocher dans le temps l'action de la subordonnée et celle de la principale (voir les exemples cités supra). ${ }^{244}$ Le même rôle revient aux locutions-corrélatifs: "nec mora", "eodem tempore" et "in ipso tempore", regroupées dans la même phrase pour préciser le sens du même cum (voir Getica, 307 - exemple commenté supra).

238 Persis est, parfois, fautivement remplacé chez Jordanès par le substantif de İère déclinaison: Persida.

239 la graphie pene pour paene est particulièrement fréquente chez Jordanès. Voir note 29.

240 le subjonctif imparfait au lieu de l'indicatif parfait des auteurs classiques (cf. Tite-Live, 2, 10, 2 ).

241 la formule plus élégante en latin "d'or" aurait été à l'aide de la conjonction $n i$.

242 septa pour saepta. La même graphie dans Getica, 213 (voir aussi note 239 etc.).

243 Voir Dag Norberg, Manuel pratique de latin médiéval, op. cit., p. 171; C. H. Grandgent, Introducción al latin vulgar, traduction de Francesco de B. Moll, Madrid, 1928, p. 95. Cf. H. Rönsch, Itala und Vulgata, op. cit., p. 431.

244 Tum et iam sont des corrélatifs spécifiques de la conjonction cum. 
Le corrélatif mox apparaît aussi pour dum suivi du plus-que-parfait du subjonctif - un seul exemple (Romana, 293). Dans un autre cas, dum construit avec le plus-que-parfait du subjonctif a le corrélatif sic (Getica, 307),

C'est toujours avec le rôle de rapprocher dans le temps l'action de la subordonnée de celle de la principale, qu'apparaît le corrélatif mox pour la conjonction postquam, dans deux cas (les subordonnées sont toutes les deux construites avec l'indicatif - voir Romana, 135, exemple commenté supra, et Getica, 146).

Nous constatons donc la répétition du corrélatif mox, pour cum et dum construits avec le plus-que-parfait du subjonctif et pour postquam suivi de l'indicatif. Mox était, nous venons de le dire, un mot précis, très répandu en latin vulgaire et chez Jordanès.

Remarquons, d'autre part, la présence des mêmes corrélatifs qu'en cas de $u b i$ et ut (mox et statim).

Nous pouvons observer aussi que locutions conjonctives n'apparaissent pas dans les propositions dépendantes indiquant l'antériorité non déterminée; nous retrouvons pourtant chez Jordanès des locutions conjonctives dans le cadre de la relation d'antériorité immédiate (ubi semel, ut primum etc.).

C) Le moment où commence l'action dans la principale est indiqué, chez Jordanès, par les conjonctions quam et postquam.

Tant quam que postquam sont très rarement utilisés par Jordanès avec cette valeur (un exemple pour chacune d'elles).

Quam et postquam (au sens de "depuis que") apparaissent chez Jordanès dans les mêmes conditions qu'en latin classique, c'est-à-dire lorsque la principale contient un adjectif numéral ordinal ou cardinal, ou bien un mot indiquant le temps (substantif, adverbe). Voir Romana, 164: "Intra enim sexagesimum diem quam caesa silua fuerat, centum sexaginta nauium classis in anchoris stetit..." (Passage copié sur Florus, 2, 2, 7); voir aussi Getica, 163: “... interiores Spanias ${ }^{245}$ introibit ${ }^{246}$, ubi saepe cum Vandalis decertans tertio anno, postquam Gallias Spaniasque domuisset, occubuit gladio ilia perforata Eueruulfi...".

On remarque que, dans l'exemple des Romana, à cause de l'influence de Florus (influencé, à son tour, par Tite-Live), c'est quam qui est employé, tandis que dans les Getica on utilise postquam, c'est-à-dire la formule plus claire et plus récente (pour le passage tiré des Getica nous ne pouvons pas établir l'influence de quelque auteur antérieur ou contemporain).

Quam est suivi de l'indicatif plus-que-parfait, pour exprimer la réalité et l'antériorité d'un fait. L'auxiliaire est au plus-que-parfait, au lieu d'être à l'imparfait, phénomène assez fréquent chez Jordanès. ${ }^{247}$

245 Pour la forme Spanias, voir note 170.

246 introibit pour introiuit.. La confusion des consonnes $b$ et $u$ est aussi fréquente chez Jordanès (voir aussi libabimus - Getica, 55; bobes - Romana, 128 etc.; voir aussi notes 73 et 84).

247 Voir Getica, 40: fuere laudati pour sunt laudati; ibid., par 65: fuerant adepti, pour erant adepti etc. Voir 
Pour ce qui est de la construction de postquam, voir le commentaire aux pages antérieures.

Le commencement de l'action de la principale peut être indiqué par des propositions relatives aussi. En voici une formule erronnée chez Jordanès: "Ac sexto quo profectus erat mensae ${ }^{248}$ (Claudius) Romae ${ }^{248}$ repedauit ${ }^{249}$ ibique defunctus est...", Rom., 260 (Cf. Orose, 7, 6, 10: "ac sexto quam profectus erat mense, Romam rediit.")

L'étude approfondie des temporelles d'antériorité nous conduit à la conclusion que le degré d'antériorité n'est déterminé ni par la conjonction et, souvent, ni par le temps utilisé dans la subordonnée, mais par divers autres facteurs, tels les adverbescorrélatifs, ou bien les adverbes employés dans le cadre des locutions conjonctives, ou bien le contexte.

Des subordonnées qu'introduisent diverses conjonctions et qui diffèrent au point de vue du temps et du mode, peuvent se situer sur le même plan temporel. Voir, par exemple: "Quod ubi... agnouerunt, magnis aggeribus eandem urbem ... struunt...", Get., 195; "Quod ubi rex ... cognouisset,... fines eius coepit praedare. ${ }^{250 ",}$ Get., 272; "Quod ut Gepidarum rex conperit..., contra filios Attilae primus insurgit...", Get., 260; "Quod cum Gratianus imperator ... conperisset,... mox ad eosc ... uenit...", Get., 141; "Quod dum Iustinianus imperator... audisset..., sic est commotus.", Get., 307; "Sed postquam ad senium peruenisset et se in breui ${ }^{251}$ ab hac luce egressurum cognusceret $^{252}, \ldots$ Athalaricum ... regem constituit...., Get., 304 etc.

Bien qu'ils jouent un rôle important dans la détermination des rapports temporels, les adverbes qui accompagnent les conjonctions d' antériorité de divers types sont assez rares. Il s' agit, d'habitude, d'adverbes qu'évitent les écrivains classiques - ilico, mox etc., ou bien l'expression employée en latin postclassique: "nec mora". L'adverbe qui sert le plus souvent à préciser le sens d'une conjonction et à établir un certain niveau d'antériorité c'est mox (soit en tant que corrélatif, soit dans le cadre de diverses locutions conjonctives).

\section{III. - LE RAPPORT DE POSTÉRIORITÉ}

Les propositions temporelles qui indiquaient la limite supérieure de la durée de l'action ou de l'événement de la principale étaient introduites, en latin cultivé classique, par les conjonctions antequam et priusquam, au sens de "avant de", "avant que".

notes 124 et 232.

248 Romae - confusion (fréquente en bas latin) entre le lieu où se déroule une action et le lieu vers lequel se dirige un mouvement. Mensae - hyperurbanisme, au lieu de mense.

249 verbe rare qui n'apparaît qu'à l'époque archaïque et tardive. Orose, 7, 6, 10, utilise rediit.

250 au sujet de la forme active praedare, voir note 87 etc.

251 au sujet de cette locution adverbiale, voir note 212 .

252 Pour cognusceret, voir note 213. 
Les modes employés dans les propositions introduites par ces conjonctions étaient l'indicatif (lorsqu'on indiquait seulement le moment temporel du fait énoncé par le verbe de la principale), ou le subjonctif (lorsqu'on ajoutait, à l'idée de temps, une nuance finale ou de possibilité - en dehors des cas où le subjonctif était réclamé par le style indirect, ou était dû à l'attraction modale). D'ailleurs, le seul fait que la proposition subordonnée exprimait une action qui n'avait pas encore eu lieu au moment où se produisait l'action de la principale, tendait à appeler le subjonctif après antequam et priusquam ${ }^{253}$ - le subjonctif utilisé comme expression du futur. Le subjonctif pour des relations purement temporelles apparaît déjà chez Lucrèce $(4,840-41)$, Cicéron, (Phil., 1, 1 etc) et se développe en latin postclassique depuis Tite-Live.

Quoique priusquam soit une conjonction plus ancienne (et préférée de Népos et César ${ }^{254}$ ), antequam la remplace, petit à petit, à l'époque classique et impériale. En bas latin, antequam était très fréquent, notamment en latin vulgaire ${ }^{255}$. Tout comme bien d'autres écrivains tardifs, Jordanès emploie beaucoup plus fréquemment la conjonction antequam que priusquam. Ainsi, priusquam n' apparaît que trois fois - d'ailleurs, les trois exemples sont regroupés au commencement des Romana, dans dés passages pour lesquels on peut établir avec précision l'influence de Florus. Voir un exemple de ce genre dans Romana, 146 - la construction grammaticale est reprise sur Florus, 1, 16, 12; un autre exemple dans Romana, 163 - passage copié sur Florus, 2, 2, 6; le troisième exemple dans Romana, 179 - passage copié sur Florus, 2, 4, 3.

Antequam est employé chez Jordanès à onze fois: il y en a cinq exemples dans les Romana et six dans les Getica. Antequam est donc quatre fois environ plus fréquent que priusquam.

Les exemples contenant antequam ne commencent qu'avec le paragraphe 333 des Romana, le final de cet ouvrage appartenant en beaucoup plus grande mesure à Jordanès (au point de vue de l'expression) que la partie du début et le milieu. Dans les Getica, antequam fait son apparition dans des phrases où l'intervention de l'auteur est évidente, par exemple: "Cuius soli terminos, antequam aliud ad medium deducamus, necesse est ... edicere.", Get., 29. Voir aussi Getica, 194: "Sed antequam pugnae ipsius ordinem referamus, necessarium uidetur edicere...".

Priusquam présente, dans tous les trois exemples, le phénomène de la tmèse, voir Romana, 146: "Nec prius finis caedibus datus, quam iugum sibi promissum Romani et duci Samnitum et hostibus reposuerunt."; mais il s'agit, comme nous venons de le dire, de phrases copiées sur Florus. Précisons toutefois que, dans deux des trois cas où apparaît prius ... quam, par le fait que le verbe principal est frappé de négation, la

253 Voir Al. Ernout - Fr. Thomas, op. cit., pp. 369-70. par. 365.

254 Voir R. Kühner - C. Stegmann, II -2, op. cit., p. 366, par. 209 , 1.

255 Cf. en ancien français:

ainz que" (voir P. Imbs, Les propositions temporelles en ancien français, Strasbourg, 1956, p. 416 sqq.) Voir Wilh. Meyer-Lübke, Grammatik der romanischen Sprachen, vol. III, op. cit., p. 650; Fried. Stolz J. G. Schmalz, Lateinische Grammatik, II -2, op. cit., p. 735. 
subordonnée temporelle indique en fait un rapport d'antériorité, et non pas de postériorité. Antequam (ou ante ... quam) n'apparaît cependant jamais chez Jordanès pour la relation temporelle d'antériorité.

La conjonction antequam présente parfois le phénomène de la tmèse, c'est-à-dire dans environ $27 \%$ des exemples. Tous les exemples de tmèse véritable se retrouvent dans les Romana; il se peut que la tmèse dans les Romana soit due à l'influence des exemples de priusquam à tmèse du même ouvrage. Voici un exemple de antequam à tmèse: “... ante ea ${ }^{256}$ uictus cupiditate pecuniis uendidit regi Vandalorum, quam in Romanorum potestatem redigeret"., Rom., 337. Dans deux autres cas, la tmèse de antequam est précisément due à l'influence du modèle suivi: voir Romana, 342, en comparaison de Marcellinus, 476 et Romana, 364, par rapport à Marcellinus, 532.

La tmèse de antequam - marque de distinction à l'époque tardive - est contrecarrée par la présence d'exemples tels:

a) Ante est parfois écrit séparément de quam, mais à la proximité de la conjonction, voir Getica, 127: "Nam maribus ferro genas secant, ut ante quam lactis nutrimenta percipiant, uulneris cogantur subire tolerantiam”. Voir en plus Getica, 186. Pour ces deux passages, les divers codices présentent l'adverbe ante séparé d'avec quam, mais occupant une position très proche du dernier.

b) dans les Romana, 358, ante est employé tant dans la principale (ou, mieux encore: au commencement des principales qui sont placées avant la même subordonnée temporelle de postériorité) qu'avant la conjonction quam: “Contra quem dum Hypatius nepus ${ }^{257}$ Caesaris egreditur, ante ab Hunnis auxiliaribus capitur et Vitaliano ... uenditur, antequam aperto proelio ... sese inimicum ostenderet." L'auteur aura eu l'intention soit d'écrire séparément les deux adverbes, oubliant par la suite son plan initial, soit - ce qui est plus probable - d'insister sur l'adverbe ante, en le répétant, pléonasme fréquent d'ailleurs en latin vulgaire à diverses époques (voir "priusquam prius", à trois fois chez Plaute; "ante - antequam" chez Varron, R. r., 2, 8, 1 et Vitruve, 2, praef., 5; voir, pour un autre niveau stylistique, la combinaison "prius - ante quam" qu'emploie Virgile, Aen., 4, 24 sqq. etc. etc.).

A la place de quam on rencontre, dans un cas, d'une manière erronée, nisi. Voir Romana, 222: “... quam ${ }^{258}$ pene $^{259}$ non ante Romanus populus adiit, nisi et suo labore uicina loca cepisset...". De telles formules apparaissent chez d'autres auteurs tardifs aussi, voir Commodien, Apol., Carm. de duobus pop., 247-48: "non ante tamen nisi..."260.

$256 e a$ - accusatif singulier (Carthaginem). La forme ea apparaît dans des codices importants tels: Heidelbergensis, Palatinus et Valenciennensis. Cependant, dans le Laurentianus, on retrouve eam.

257 Pour nepus, voir note 99.

258 quam au lieu de quod (se rapportant à "regnum").

259 de nouveau l'adverbe pene, avec la graphie vulgaire $e$.

260 Voir une expression plus compliquée chez Calpurnius Siculus, Buc., 1, 87-88: "nec prius - nisi cum". Pour 
Précisons que, dans de tels exemples, à cause de la négation dans la principale, la subordonnée introduite par nisi indique un rapport temporel d'antériorité.

Quant aux modes utilisés, priusquam est construit, conformément à l'usage classique, dans un cas avec l'indicatif - étant énoncée seulement la relation temporelle entre la proposition dépendante et la proposition corrélative (voir l'exemple déjà cité, Romana, 146). Dans les deux autres cas, priusquam est suivi du subjonctif, à l'idée de temps venant s'ajouter une nuance de possibilité ou de but. Voir Romana, 163: “... statimque ac sine mora ${ }^{261}$ Hyeronem Syracusanum tanta celeritate deuicit, ut ille ipse prius se uictum, quam hostem uideret, fateretur." Voir aussi Romana, 179.

En ce qui concerne la conjonction antequam, celle-ci est, d'ordinaire, suivie du subjonctif, même lorsque seul le moment temporel est indiqué. Voir Romana, 333: “... obitumque Attilae et Zenonis Isauri interitum, antequam moriretur ${ }^{262}$, felix conperit $^{263}$ infelicium." En traduction: "Avant de mourir, (Marcien) a eu le bonheur de voir la fin de ses ennemis: Attila et Zénon l'Isaurien." Voir aussi Romana, 337.

Mais, assez souvent, le subjonctif peut être motivé par l'existence d'une nuance finale, par exemple: “... amputatisque eorum capitibus ante eos fecit ${ }^{264}$ imperium perdere quam haberent.", Rom., 364. Voir également Romana, 358 (exemple cité à la page antérieure), Getica, 29 etc.

L'indicatif apparaît après antequam une seule fois - voir Romana, 342: "Sed uolente deo ante inflatus crepuit ${ }^{265}$ quam penitens ${ }^{266}$ stare potuerat." Mais chez Marcellinus (476) on trouve potuerit ${ }^{267}$.

Le seul exemple d'indicatif après antequam se retrouve donc chez Jordanès dans son premier ouvrage.

La fréquence de l'indicatif dans les subordonnées introduites par antequam est, pourtant, très faible.

L'emploi du subjonctif pour indiquer une relation purement temporelle pénètre dans la littérature dès la fin de l'époque républicaine et s'accentue dans les périodes suivantes. En bas latin, divers écrivains évitent systématiquement l'indicatif: Ambro-

d'autres exemples de "non ante - nisi" à l'époque tardive, nous renvoyons à J.B. Hofmann - A. Szantyr, II - 2, op. cit., p. $596^{1}$

261 "statim ac sine mora" - pléonasme, S'y ajoute le syntagme "tanta celeritate". Pour des répétitions de ce genre, voir note 111 .

262 Dans le Codex Palatinus (IX ${ }^{\mathrm{e}}$ siècle) - la forme "moreretur".

263 De nouveau, la graphie conperit.

264 "oes fecit imperium perdere" - construction propre à la langue archaïque et au latin "de la décadence" (pour d'autres exemples de facio accompagné de l'infinitif, voir R. Iordache, L'infinitif dans les oeuvres de Jordanès, op. cit., pp. 130-131.)

265 crepuit - vulgaire, au lieu de corruit.

266 penitens pour paenitens. Le participe présent sans le diphtongue -ae-apparaît encore une fois, dans les Getica, 225.

267 Dans le Codex Laurentianus (pour Jordanès), $\mathrm{XI}^{\mathrm{e}}$ siècle, apparaît la forme potuerit. 
se, Jérôme, Minucius Félix, Sulpice Sévère, Victor de Vite, Cassiodore. L'indicatif est presque inexistant dans Vulgata, Gesta Francorum etc. (voir Vulgata, Ioh., 8, 58: "antequam Abraham fieret, ego sum.").268

Le subjonctif immotivé apparaît chez Jordanès seulement après antequam, conjonction fréquente en latin vulgaire et chez Jordanès.

Quant à l'emploi des temps, nous constatons:

1. l'apparition fréquente de l'imparfait du subjonctif, auquel correspond dans la principale soit: a) le parfait de l'indicatif - la situation habituelle (voir Romana, 333, 337, 364, Getica, 238, 297 - pour antequam; Romana, 163 - pour priusquam), soit: b) le présent historique (Romana, 358 - dans la principale qui régit antequam), soit: c) l'imparfait de l'indicatif (voir Getica, 186 - dans la principale régisssant antequam).

2. Assez souvent, antequam est suivi du présent du subjonctif qui correspond au véritable présent de l'indicatif, ou au présent du subjonctif exprimant le but, voir Getica, 29 (exemple ci-dessus), Getica, 127 et 194.

3. Dans un seul exemple, on retrouve le parfait de l'indicatif - après priusquam. Le verbe de la principale est au parfait de l'indicatif-Romana, 146 (le verbe principal est frappé de négation).

4. Une seule fois apparaît, après antequam, le plus-que- parfait de l'indicatif temps très rare dans ce type de subordonnées, durant toute la latinité269. C'est toujours le parfait de l'indicatif qui apparait dans la principale: "Sed uolente deo ante inflatus crepuit, quam penitens stare potuerat", Rom., 342. En de telles situations, le plus-queparfait n'a pas d'aspect de perfectum, car il substitue un imparfait (Cfr., pour l'utilisation du plus-que-parfait à la place de l'imparfait chez Jordanès: “... ita sunt proeliati, ut ... non remansissent...", Get., 276).

5. Un exemple de plus-que-parfait du subjonctif, après "non prius - quam", Rom., 179. Un autre exemple de subjonctif plus-que-parfait après "non ante - nisi", Rom., 222.

D'autre conjonctions qui, en latin cultivé classique, indiquaient la postériorité de l'action de la temporelle c'étaient: dum, donec et quoad avec le sens de "jusqu'à". 270

268 Certaines langue romanes présentent, pour les conjonctions de postériorité, une évolution semblable à celle de antequm. En français moderne, la locution avant que est construite uniquement avec le subjonctif; de même en italien les locutions avanti che et prima che; cfr. en roumain: (mai) inainte ca + subjonctif.

269 Chez Cicéron, l'indicatif plus-que-parfait est attesté une seule fois - voir S. Sciuto, Grammatica della lingua latina, Torino, 1967, p.988. Voir quelques exemples d'indicatif plus-que-parfait, le long de la latinité, recueillis par R. Kühner - C. Stegmann, II -2 op. cit., p. 371, par. 209, 5, c.

270 Indiquant la limite de l'action de la principale, le laps de temps qu'accorde la subordonnée à l'action de la principale, ces conjonctions au sens de "jusqu'à" sont spécifiques du rapport de postériorité, et non pas du rapport de simultanéité (comme le soutiennent de nombreux chercheurs). 
La manière dont ces conjonctions étaient construites, en latin classique, était la suivante:

1. indicatif-a) présent, lorsque la principale contenait un présent, un futur ou un impératif;

b) parfait ou présent historique, si dans la principale il y avait un temps passé;

c) futur antérieur, correspondant à un futur ou à un impératif dans la proposition principale.

ou:

2. subjonctif - a) présent, correspondant aux mêmes temps de la principale que l'indicatif présent;

b) imparfait.

L'indicatif était employé lorsque dans la temporelle on énonçait un fait réel ou considéré comme tel (il pouvait y avoir une nuance d'intention, sans qu'on insistât la-dessus). La conjonction avait le sens de "jusqu'au moment où".

Le subjonctif était utilisé lorsque la subordonnée renfermait une idée nettement dessinée de but, intention ou attente. La conjonction avait le sens de "jusqu'à ce que". Avec Tite-Live, le subjonctif entre dans la sphère de l'indicatif et cette tendance s'accentue aux siècles suivants; dans les écrits de divers auteurs de l'époque postclassique et tardive, ce phénomène apparaît souvent - Tacite, Avit etc. ${ }^{271}$

Parmi les conjonctions anciennes et classiques ayant le sens de "jusqu'à", seules dum et donec apparaissent chez Jordanès; chacune d'entre elles est employée avec cette valeur à deux fois. L'utilisation peu fréquente de dum s'explique par le fait que, chez Jordanès (tout comme chez d'autres auteurs tardifs - Grégoire de Tours et même Avit), dum a très souvent le sens de "pendant que", remplaçant cum. Donec au sens de "jusqu'à", quoique fréquent chez certains auteurs tardifs (Cyprien, Prudence, Peregrinatio Aetheriae ${ }^{272}$ ), est rare chez Jordanès. Rappelons, à cette occasion, que donec au sens de "tant que" est employé, chez Jordanès, une seule fois. D'autre part, compte tenu du fait que priusquam - conjonction propre au rapport de postériorité - n'apparaît que trois fois et que antequam n'est guère trop fréquent, nous pouvons conclure que l'auteur n'a pas eu l'intérêt à construire ce type de subordonnée.

Dum apparaît dans un exemple avec valeur purement temporelle: “... regnumque cognatis, usque dum ille (Gratianus) Theodosium ordinasset, fideliter uiriliterque seruauit (Dominica Augusta)", Rom., 314273. Dans l'autre exemple, dum a une valeur temporelle-finale ou, plus exactement, temporelle-consécutive-finale: “... adeoque

271 Voir, là-dessus, R. Kühner-C. Stegmann, II -2, op. cit., p. 380, par. 210, point 8.

272 Voir J.B. Hofmann - A. Szantyr, II-2, op. cit., p. 629. D'autres auteurs, tels Ammien ou Benoit de Nurse, ignorent donec, Chez Avit, donec au sens de "jusqu' a" est plus rare que dum (voir $\mathrm{H}$. Goelzer, Le latin de Saint Avit, op. cit., p. 342).

273 La nature des relations entre l'empereur Valens et le père du futur empereur Théodose $\mathrm{I}^{\mathrm{er}}$ nous prouve qu'il s'agit, dans ce passage, de dum à valeur purement temporelle. 
moratus (est) ostes ${ }^{274}$, dum exercitus omnis euaderet", Rom., 166 (passage copié, au point de vue de la construction grammaticale, sur Florus, 2, 2, 13).

Donec est construit, dans un exemple, avec l'indicatif parfait: "Tarquinii tamen diu dimicauerunt, donec Arruntem filium regis manu sua Brutus occidit superque ipsum mutuo uulnere expirauit ${ }^{275}$..." (passage copié sur Florus, 1, 10, 8, à une seule différence près: chez Florus, à la place de diu, il y a "tam diu". Dans ce cas, le corrélatif de donec est, chez Jordanès, l'adverbe diu).

Dans un autre passage (copié sur Florus, 2, 6, 17), donec est suivi du subjonctif: "Itaque duo maximi exercitus caesi ad hostium satietatem, donec Annibal ${ }^{276}$ diceret militi suo "parce ferro", Rom., 188.

Observons donc que tant dum que donec sont construits - une fois chacun - avec le subjonctif, sans égard pour les règles de la syntaxe classique, voir les exemples cités, Romana, 314 (voir aussi note 274) et Romana, 188.

Pour ce qui est de l'emploi des temps, on ne remarque comme dérogation aux normes que l'exemple des Romana, 314 (voir ci-dessus). Dans ce cas, le subjonctif plus-que-parfait est utilisé à la place du subjonctif imparfait ou, plus exactement, à la place de l'indicatif parfait - si nous nous en tenons aux règles du latin classique. Le subjonctif plus-que-parfait ne fait son apparition dans ce type de subordonnée (après dum, donec et quoad avec le sens de "jusqu'à") que dans la période postclassique (avec Tite-Live) et n'est que très rarement attesté durant toute la latinité ${ }^{277}$ Le subjonctif plus-que-parfait est un peu plus souvent attesté, dans les propositions introduites par antequam et priusquam, dès l'époque classique. ${ }^{278}$

Ce qu'il y a d'intéressant c'est que tous les exemples de dum et donec (au sens de “jusqu'à") sont attestés dans les Romana. Remarquons, en revanche, l'emploi plus fréquent de antequam dans les Getica.

Les antécédents de ces conjonctions sont nombreux, par rapport au nombre des exemples. Dans un cas, dum forme locution avec l'adverbe usque (Rom., 314). Dans l'autre cas, dum a pour corrélatif l'adverbe adeo (Rom., 166). Si usque joue, à toutes les époques, le rôle d' antécédent tant pour dum au sens de "aussi longtemps que" (voir aussi notre commentaire, page 41 de la première partie de cette étude), que pour $d u m$ au sens de "jusqu'à", adeo, lui, ne fonctionne comme antécédent que pour dum, donec et quoad au sens de "jusqu'à" - et ce, dès l'époque préclassique (voir Plaute, Amph., 472: "adeo usque ... dum"; Asin., 328: "adeo usque dum..."; Rud., 811: "usque adeo

274 l'omission de l'aspirée dans le cas du substantif hostis est fréquente chez Jordanès (voir également Romana. 96,97 etc.).

275 expirauit, pour exspirauit (même graphie chez Florus).

276 Annibal, pour Hannibal. La forme sans aspirée est très fréquente dans les écrits de Jordanès (voir aussi Romana, 181, 182, 183, 188 etc. etc.).

277 Voir R. Kühner - C. Stegmann, II - 2, op. cit., p. 381, par. 210, 8.

278 Voir R. Kühner - C. Stegmann, II -2, op. cit., pp. 370-371, par. 209, 5, b. 
donec..."; Caton, R.r., 106, 2: "usque adeo donec"; ibid., 95, 1: "usque adeo dum" etc.).

Donec est précédé par le corrélatif diu - voir Romana, 123 (exemple ci-dessus).

Tant les subordonnées introduites par antequam et priusquam, que les propositions introduites par dum et donec (au sens de “jusqu'à") sont généralement placées après la proposition principale. Parfois, les subordonnées sont intercalées entre le sujet et le prédicat de la principale, ou bien entre le complément d'objet direct (et son groupe) et le prédicat de la principale (voir Getica, 29); d'autres fois, la subordonnée précède la principale (surtout lorsqu'il ne s'agit pas de narrations historiques), voir, par exemple, Getica, 194: "Sed antequam pugnae ipsius ordinem referamus, necessarium uidetur edicere...".

Entre toutes les conjonctions indiquant l'antériorité de la principale par rapport à la subordonnée, seule la conjonction donec introduit deux verbes dans une phrase, voir Romana, 123. Les autres conjonctions, même antequam, n'introduisent qu'une seule proposition temporelle - encore un indice prouvant que Jordanès n'a pas voulu donner ampleur à ce type de subordonnée.

Une autre conjonction ayant, chez Jordanès, le sens de "jusqu'à" c'est quate$n u s^{279}$.

A l'époque tardive, quatenus est souvent utilisé dans les ouvrages à caractère scientifique et technique, ainsi qu'en style ecclésiastique et administratif. ${ }^{280}$

La conjonction quatenus est assez fréquente chez Jordanès - treize exemples. Soulignons que la plupart des exemples se trouve dans les Getica (neuf) et que, de plus, c'est toujours dans les Getica que cette conjonction acquiert les plus variées et les plus complexes valeurs syntactico-sémantiques; d'ailleurs, comme nous l'avons dit, cet ouvrage présente davantage un style propre à l'auteur dont nous nous occupons.

On pourrait se demander: pourquoi Jordanès utilise-t-il la conjonction quatenus? C'est, croyons-nous, puisque quatenus offrait l'avantage d'une plus grande précision sémantique (ainsi que d'un corps phonétique plus grand), en comparaison de bien d'autres conjonctions anciennes, surchargées de valeurs, usées et dépourvues d'expressivité (telles ut, cum, quoniam, qua et quam). D'autre part, quatenus - conjonction plus récente et ayant un nombre assez limité de valeurs sémantiques (du moins aux époques classique et postclassique) - n'avait pas besoin de corrélatif.

Quatenus a, chez Jordanès, les valeurs suivantes: a) finale (le plus souvent); consécutive-finale: b) complétive; c) temporelle; d) causale.

En général, Jordanès - tout comme la plupart des juristes et des ecclésiastiques de l'époque tardive - préfère employer quatenus suivi du subjonctif, à la place de $u t$

279 Dans sa dissertation (op. cit.), Fr. Werner ne dit rien au sujet de quatenus temporel.

280 Voir Ed. Wölfflin, Quatenus, en "Archiv für lateinische Lexicographie und Grammatik", vol. V - 1, Leipzig, 1888, pp. 399-414. 
introduisant une proposition finale, une consecutive, une complétive (ou une proposition sujet). Quoiqu'il en soit, les valeurs sémantiques de cette conjonction présentes chez Jordanès sont de celles assez récentes. Il s'agit, bien sûr, de sens figurés de la conjonction quatenus.

Quatenus temporel apparaît très rarement chez Jordanès. Chez cet écrivain, quatenus introduit des propositions temporelles de postériorité; la conjonction a le sens "jusqu'à".

Voici un exemple où quatenus a valeur temporelle: "Hic (Tarquinius Priscus) et senatus maiestatem numero ampliauit et centuriis tribus auxit, quatenus Actius Neuius $^{281}$ numerum augeri prohibebat, uir. summus augurio.", Rom., 99.282

Dans ce fragment (copié d'ailleurs sur Florus, $1,5,2$ ) ce qu'il y a de surprenant c'est le temps du verbe prohibere, le parfait de l'indicatif ayant été, certes, préférable. Jordanès (et, probablement, Florus aussi) aura hésité entre les divers sens de quatenus ("dans la mesure où", "pendant que" et "jusqu'à"), ce qui a déterminé l'emploi de l'imparfait.

Voici un autre exemple où quatenus a une valeur temporelle-finale: "Gyzericus etenim Vandalorum rex suis cum muneribus ad ista commitenda inlicuit ${ }^{283}$, quatenus ipse Leonis uel ${ }^{284}$ Zenonis insidias, quas contra eum ${ }^{285}$ direxerant, praecauerat...", Get., 244. La traduction de cette phrase est: "Car Gyzéric, roi des Vandales, engagea, par ses cadeaux, celui-ci (Eurichus) à faire ces conquêtes (Arles et Marseille), jusqu' à ce que lui-même se mette à l'abri des pièges que Zénon et Léon avaient ourdis contre lui."

Le sens "jusqu'à" de quatenus était probablement fréquent en latin de chancellerie et dans les ouvrages scientifiques de l'époque tardive, bien que les exemples dont nous disposons soient peu nombreux ${ }^{286}$. En voici un, tiré de Scholia Ciceronis Bobiensia (IVe siècle), p. 132, 10, St.: "Quod Caesar eatenus ante portas ... permanserit, quatenus in exilium Cicero discederet." On remarque l'emploi du subjonctif dans la subordonnée introduite par quatenus, tout comme dans l'exemple des Getica.

Rappelons que, chez Cicéron déjà, on retrouve le sens: "jusqu'à quand"? ("combien de temps"?), pour introduire des principales interrogatives et des complétives interrogatives indirectes. Chez Cicéron, les temporelles de postériorité introduites par quatenus sont pourtant inexistentes.

281 Actius Neuius, pour Attius Nauius.

282 Ed. Wölfflin considère cet exemple (Romana, 99) comme causal (op. cit., p. 406).

283 inlicuit au lieu de illexit.

284 uel au lieu de -ue.

285 eum - le pronom démonstratif est utilisé ici d'une manière erronée, à la place du pronom réfléchi.

286 Au sujet de la rareté des exemples de quatenus au sens de "jusqu'à", voir Ed. Wölfflin, op. cit., p. 404. En plus, Ed. Wölfflin hésite quant au sens des exemples qu'il présente.

La plupart des grammaires et des dictionnaires importants de la langue latine excluent le sens temporel de "jusqu'à" dans le cas de quatenus. 
Mentionnons que chez Jordanès le sens de "jusqu'à" est rendu, en premier lieu, par les conjonctions dum et donec, mais peu souvent (à deux fois chacune d'elles).

Par rapport au nombre d'exemples de dum et donec (au sens de “jusqu'à"), Jordanès a fréquemment recours à quatenus (cette conjonction étant plus récente et mieux précisée au point de vue sémantique). Ajoutons que tous les exemples de dum et donec introduisant des temporelles de postériorité apparaissent dans les Romana, tout comme un exemple de quatenus, tandis que, dans les Getica, l'unique conjonction ayant le sens de "jusqu'à" c'est quatenus.

L'exemple de quatenus des Getica est d'autant plus important que les attestations de quatenus au sens de "jusqu'à" sont peu nombreuses en bas latin.

En résumant on peut donc dire que bien des conjonctions temporelles utilisées par Jordanès se retrouvent en latin classique (cum, dum, donec, quamdiu, $u b i, u t$, postquam, quam, antequam et priusquam - à savoir dix conjonctions anciennes). A côté de ces conjonctions fait son apparition une conjonction ancienne, importante par son emploi temporel, mais que l'usage classique évite - quando, ainsi qu'une série de conjonctions et de locutions conjonctives, la plupart postérieures à l'époque classique: quousque, mox ubi, cum mox, mox, quod et tantum quod, non ante - nisi, quatenus (énumérées en fonction du rapport temporel entre la subordonnée et la principale).

Certaines conjonctions classiques acquièrent des sens nouveaux ou sont construites d'une manière nouvelle; parfois, tant le sens que la construction sont étrangers à l'usage classique. Ces phénomènes, ainsi que la présence de conjonctions et de locutions conjonctives nouvelles modifient la fréquence d'apparition de certaines conjonctions dans le texte, par rapport à l'usage classique. Ainsi dum, dans ses diverses acceptions, dépasse, au point de vue de la fréquence, cum et devient, de la sorte, la plus fréquente conjonction temporelle chez Jordanès.

Les conjonctions classiques le plus souvent remplacées sont: cum, ut et priusquam.

Il est intéressant de dresser la liste de toutes les conjonctions temporelles qui apparaissent chez Jordanès, par ordre de leur fréquence: la situation statistique ressemble davantage à celle du latin vulgaire tardif qu'à celle du latin classique. Ainsi $d u m$ (répandue en latin vulgaire aux $\mathrm{V}^{\mathrm{e}}$ et $\mathrm{VI}^{\mathrm{e}}$ siècles et conservée dans certaines langues romanes) est de loin la plus fréquente conjonction temporelle utilisée par Jordanès. Suivant, par ordre décroisssant: cum, ubi, postquam, quando, mox, ut, antequam, priusquam, donec - les deux dernières n'apparaissant que dans trois exemples; quod et quatenus font leur apparition par deux fois, quamdiu, quousque et nisi-une seule fois. De cette énumération on peut constater aussi l'existence de plusieurs conjonctions synonymes pour des temporelles du même type, ce qui donne de la variété stylistique à l'écriture de Jordanès.

L'influence du latin vulgaire sur Jordanès s'est exercée, dans le domaine de la subordonnée temporelle, de diverses manières dont nous citons ici les plus importantes: 
I. - Certaines conjonctions anciennes, usées, ont cédé tant leur sens que leur construction, à d'autres conjonctions ou locution conjonctives, étrangères à l'usage classique. Ainsi, quando remplace, partiellement, cum explicatif- temporel et cum itératif. Mox et quod se substitue, en grande mesure, à ut temporel; quatenus remplace quoad et donec; quousque remplace quamdiu; cum mox et tantum quod se substituent à ut primum; non ante - nisi, à non prius-quam.

Parmi les conjonctions "nouvelles", la plus fréquente est quando, conjonction très employée en latin vulgaire tardif, conservée dans toutes les langues romanes (voir, d'ailleurs, note 79).

On observe également que les locutions conjonctives et les conjonctions nouvelles apparaissent, en premier lieu, pour exprimer le rapport d'antériorité et, en second lieu seulement, pour les rapports de simultanéité et de postériorité.

L'écrivain semble avoir fait des efforts notamment pour exprimer d'une manière aussi précise et nuancée que possible le rapport d'antériorité.

II. - Parfois, les conjonctions anciennes ajoutent un certain sens et imposent une certaine construction à des conjonctions appartenant déjà au latin classique, mais qui avaient des valeurs moins nombreuses. C'est ainsi que dum reprend la construction de cum narratif d'antériorité et, dans une certaine mesure, le sens aussi; dum remplace, de même, cum "uere temporale".

III. - D' autres fois, les conjonctions anciennes ne cèdent que leur construction à des conjonctions à sens proche, présentes déjà en latin classique. Ainsi, sous l'influence de cum narratif de simultanéité, dum et $u t$ sont suivis du subjonctif imparfait. Dum suivi du subjonctif imparfait (très rarement du présent du subjonctif) devient même plus fréquent que cum narratif de simultanéité.

Sous l'influence de cum narratif de simultanéité, postquam est construit avec le subjonctif imparfait.

Par analogie avec la construction de cum narratif d'antériorité, ubi, mox et postquam sont suivis du subjonctif plus-que-parfait.

L'extension du subjonctif après ces conjonctions n'est pas égale chez Jordanès. Ainsi, le subjonctif plus-que-parfait est particulièrement fréquent après postquam (dont le sens de "après que" s'accordait avec l'utilisation du plus-que-parfait) et plus rare après mox et, surtout, $u b i$ : il s'agit là d'une barrière que s'impose Jordanès aux dérogations à la norme classique, concernant soit le sens, soit la construction. Rappelons, dans le même ordre d'idées, que dum suivi du subjonctif plus-que-parfait est beaucoup plus rare que dum suivi du subjonctif imparfait.

L'apparition du subjonctif plus-que-parfait après une série de conjonctions et l'emploi presque courant du subjonctif imparfait après d'autres conjonctions a conduit, finalement, à la modification du sens de certaines conjonctions. Ainsi, l'emploi du subjonctif imparfait après postquam modifie le sens de cette conjonction la déplaçant de la sphère de l'antériorité non déterminée dans celle de l'antériorité récente (avec le sens de: "dès que", "lorsque", "comme"). 
Si grand nombre des aspects que nous venons de passer en revue apparaissent assez souvent chez d'autres auteurs aussi, ubi non itératif suivi du subjonctif plus-queparfait, dum et mox construits de la même manière et postquam accompagné du subjonctif plus-que-parfait et imparfait sont très rarement attestés avant Jordanès. Les écrits de Jordanès sont donc de précieux enregistrements de faits de langue évités par les écrivains contemporains et, à plus forte raison, par les écrivains antérieurs. Certains de ces aspects sont même assez frequents chez Jordanès, par exemple la construction de postquam avec le subjonctif et la construction similaire - bien que plus récente et plus rarement attestée - de $d u m$, et pourraient constituer un indice de l'utilisation plus fréquente, de cette manière, de postquam et $d u m$ en latin vulgaire au VIe siècle.

Il convient de faire remarquer que tous les aspects déjà mentionnés, rarement attestés avant Jordanès, apparaissent dans le cadre du rapport d'antériorité, rapport dont Jordanès précise les nuances de diverses manières, selon les nécessités et selon les connaissances de l'écrivain.

D'autre part, si l'on dressait la statistique des aspects étrangers à l'usage classique, qui apparaissent dans le cadre de chaque rapport, on constaterait que les plus nombreuses "innovations" concernent l'expression du rapport d'antériorité. Cependant, au point de vue quantitatif, les exemples d'aspects "nouveaux" répérables dans le cadre du rapport de simultanéité sont plus nombreux que ceux que présente le rapport d'antériorité: ce fait est dû, premièrement, à l'utilisation fréquente de dum avec le subjonctif imparfait (d'après le modèle de cum narratif).

Le processus de disparition de certaines conjonctions conduit donc, d'une part, à: I. l'apparition et la consolidation de conjonctions et locutions conjonctives nouvelles, et, d'autre part, à II. l'attribution de sens et des constructions nouveaux à des conjonctions déjà connues à l'usage classique, ou bien à: III. la consolidation de certains sens des conjonctions anciennes.

Ainsi, la disparition de $u t$ et l'apparition assez rare de $u b i$ conduisent à l'utilisation de la conjonction mox ou de la locution cum mox, ainsi qu'à l'attribution du sens "dès que" à la conjonction quod. L'emploi rare de quamdiu a eu pour résultat l' apparition de quousque ("aussi longtemps que"). L'emploi rare de dum et donec avec le sens de "jusqu'à", de même que l'absence de quoad justifient l'apparition de quatenus (="jusqu'à")

La conjonction qui, chez Jordanès, a repris la plupart des valeurs de cum c'est dum (la conjonction temporelle la plus fréquente et, en même temps, celle qui présente le plus grand nombre de dérogations aux normes classiques). Suivent, par ordre décroissant, quando et postquam.

Cette reprise de diverses valeurs de la conjonction cum s'est effectuée, certes, suivant le sens fondamental de chaque conjonction. Ainsi, dum (ancienne forme d'Instrumental, tout comme $\mathrm{cum}^{287}$ reprend, parfois, le sens explicatif-qualificatif-temporel "pendant que" de cum narratif, la rare utilisation de cum narratif contribue à raf- 
fermer le sens explicatif-temporel (non qualificatif) "pendant que" de dum suivi de l'indicatif et du subjonctif. En revanche, le sens "après que" de dum (suivi du subjonctif plus-que-parfait) est rare.

Le plus fréquent des aspects mentionnés aux points I, II et III (voir ci-dessus) est "La consolidation de certains sens des conjonctions anciennes", réalisée par le transfert de certaines constructions des conjonctions tombées en désuétude vers d'autres conjonctions à sens similaires, parfois même identiques. Ce procédé fait de Jordanès sinon un écrivain voulant imiter le style classique, du moins un écrivain qui commet plutôt des "fautes légères" que des "fautes graves".

D'autre part, au sujet des situations mentionnées aux points I et II, sont plus fréquentes celles inclues sous le point $\mathrm{I}$, à savoir 'L'apparition et la consolidation des conjonctions nouvelles". 288

IV. - Un autre domaine où l'on constate des dérogations aux normes classiques c'est celui des modes:

A. L'utilisation erronée du subjonctif, sans qu'il y soit question du transfert du modèle de construction de quelque conjonction. C'est bien le cas de ubi et quando itératifs, de antequam et non ante-nisi, de même que le cas de dum et donec au sens de 'jusqu'à".

Parfois, on retrouve l'aspect classique joint à l'aspect erroné - la même conjonction étant construite avec les deux modes, dans la même phrase -; c'est le cas de $u b i$ et quando itératifs.

B. Très rarement, on rencontre la situation inverse - l'indicatif à la place du subjonctif. Ainsi, cum narratif est construit avec le présent historique.

V. - On remarque, de même, dans les subordonnées temporelles chez Jordanès, la transgression assez fréquente des règles de la Concordance des temps. Sont en désaccord avec les normes: le parfait du subjonctif dans la subordonnée itérative, le plus-que-parfait de l'indicatif après antequam, le plus-que-parfait du subjonctif après dum (="jusqu'à"), l'imparfait de l'indicatif dans la subordonnée introduite par quatenus (=“jusqu'à").

Les locutions conjonctives, bien que très fréquentes à l'époque tardive et en latin vulgaire notamment, sont très rares chez Jordanès. On devrait chercher l'explication de ce phénomène dans le grand nombre de conjonctions “nouvelles" qu'emploie l' auteur. Cependant, en latin tardif vivant on retrouve des adverbes même à côté des conjonctions nouvelles, vigoureuses, monosémiques dans la plupart des cas. On peut dire que Jordanès, pour ce qui est de la fréquence des locutions conjonctives, garde une ligne de milieu entre le latin classique et le latin vulgaire.

287 Voir, là-dessus, R. Iordache, ¿"Cum" temporal o "cum" explicativo?, op. cit., pp. 266-68.)

288 Par conjunctions "nouvelles" on entend, d'habitude, les conjonctions apparues dans les periodes ultérieures à la période "d'or". Parfois, nous nous rapportons à un certain sens, étranger à l'usage classique (voir le sens temporel de quando par rapport à son sens causal). 
Chez Jordanès, seules les conjonctions anciennes et à plusieurs valeurs, parfois ayant un corps phonétique réduit, entrent dans des locutions conjonctives. Presque tous les exemples de locutions apparaissent dans le cadre du rapport d'antériorité (récente). Ainsi, on rencontre: ubi semel, ut primum, mox ubi, cum mox, tantum quod.

La conjonction dum n'apparaît dans des locutions que lorsqu' elle a le sens: “aussi longtemps que" et "jusqu'à" - et, chaque fois, avec l'adverbe usque: deux exemples pour le sens de "aussi longtemps que" (un taux assez élevé, vu le nombre total des exemples où dum a cette acception: cinq) et un exemple pour le sens "jusqu'à" (de fait, $50 \%$ des exemples). En revanche, dum au sens de "pendant que" est précisé seulement par son corrélatif et ce, très rarement (voir ci-dessous notre commentaire).

Les corrélatifs sont assez fréquents et précisent d'habitude le sens de conjonctions anciennes et à plusieurs valeurs. Donec a - dans l'unique exemple où son sens est "aussi longtemps que" - pour corrélatif l'adverbe tamdiu. Dum au sens de "pendant que" est, à deux fois, en corrélation avec l'adverbe ilico. Vbi et ut apparaissent en corrélation avec mox (un exemple chacun); même situation pour cum "temporel proprement dit". Vbi de simultanéité a, dans un cas, le corrélatif tunc. Cum narratif d'antériorité (suivi du subjonctif plus-que-parfait) a pour corrélatifs: eodem tempore, nec mora, in ipso tempore (agglomérés dans une seule phrase), ou: statim, mox, iam, tum (dans des exemples différents), ainsi que tandem; dum d'antériorité (construit avec le subjonctif plus-que-parfait) présente, dans un exemple, le corrélatif mox et, dans un autre exemple, le corrélatif sic. Postquam a pour corrélatif, par deux fois, mox. Dum au sens de "jusqu'à" est une fois précisé à l'aide de l'adverbe adeo et donec (= "jusqu'à"), à l'aide de l'adverbe diu.

Les conjonctions étrangères à l'usage classique qui apparaissent avec des corrélatifs sont: quando, ayant pour corrélatif tunc (deux exemples), mox en corrélation avec ilico (six exemples).

On remarque donc:

a) l'apparition de corrélatifs (adverbes ou locutions adverbiales) étrangers à l'usage classique: ilico, mox, nec mora etc.

b) l'agglomération de marques visant à préciser le sens d'une conjonction - soit l'apparition d'un groupe de corrélatifs, soit l'éclaircissement d'une locution à l'aide d'un corrélatif (c'est ainsi que ut primum est précisé par ilico, ubi semel, par statim, cum mox, par statim aussi.

c) le plus fréquent corrélatif c'est ilico, que suit mox.

d) le plus fréquent adverbe (vulgaire) en double hypostase (c'est-à-dire de corrélatif et précédant une conjonction avec laquelle il forme une locution conjonctive) c'est, cependant, mox.

e) la présence de diverses expressions (surtout de "nec mora"), ainsi que des adverbes di- et trisyllabiques (utilisés, parfois, en latin classique, comme tamdiu, mais tardifs, pour la plupart, par exemple: ilico), à la place des corrélatifs anciens, à corps phonétique réduit. 
On rencontre, certes, chez Jordanès, bien des aspects classiques aussi: la "Règle du présent" dans le cas de dum (= "pendant que") est particulièrement familière à notre auteur. L'indicatif présent est, d'ailleurs, plus fréquent dans les propositions introduites par dum (="pendant que") que le subjonctif imparfait.

Cum narratif est, en général, suivi du subjonctif imparfait et plus-que-parfait. Très rarement, il apparaît avec d'autres temps du subjonctif, ou avec l'indicatif.

Certaines dérogations témoignent des efforts que fait l'écrivain pour s'exprimer élégamment, par exemple la construction de dum, quamdiu et quousque (toutes ayant le sens de "aussi longtemps que") avec l'imparfait du subjonctif.

La plupart des dérogations se trouve dans les Getica. On y retrouve non seulement beaucoup de dérogations aux normes classiques, mais encore des constructions plus variées et plus hardies.

Nous concluons en soulignant que les Romana et les Getica sont importantes aussi par le fait qu'ils enregistrent des aspects du latin vulgaire ou du style de chancellerie rarement attestés chez les écrivains antérieurs ou contemporains. Citons, à cet égard: ubi non itératif accompagné du subjonctif plus-que-parfait, dum et mox construits avec le même mode et temps (le subjonctif plus-que-parfait), postquam suivi du subjonctif plus-que-parfait et imparfait, quatenus temporel au sens de "jusqu'à".

\section{Povzetek \\ OPOMBE K ČASOVNEMU ODVISNIKU V KLASIČNI IN KASNI DOBI LATINŠČINE: JORDANES}

Študija temelji na načelih historične sintakse in skuša predstaviti različno grajene časovne odvisnike skozi stoletja žive latinščine; predvsem se ukvarja $\mathrm{z}$ rabo časovnih odvisnikov klasične dobe in v pozni latinščni. Popravlja in dopolnjuje več teorij, ki načenjajo rabo veznikov v klasični dobi.

Za Jordanesa, avtorja s srede 6 . stoletja našega štetja, se ugotavlja, da se nekateri vezniki izgubljajo. To pa pogojuje pojav in uveljavljanje novih veznikov in vezniških izrazov, nadalje, dodajanje novih funkcij že iz klasične rabe znanim veznikom in, končno, utrditev nekaterih pomenov starih veznikov.

Študija ugotavlja tudi druge kršitve klasične norme $\mathrm{v}$ rabi naklonov in časov, tako pri sosledici časov; kršitve je mogoče ugotoviti tako pri rabi starih, znanih veznikov kot pri rabi novih.

Vezniške konstrukcije so $v$ kasni latinščini in tudi v vulgarni latinščini pogostne; vendar pa so pri Jordanesu redke, saj se drži avtor zlate sredine med rabo v klasičnem jeziku in tisto v ljudskem govoru kasne dobe.

Soodnosni zaimki so pogostni: navadno pojasnjujejo stare veznike, ki imajo največ vrednosti. Posebej se omenja: a) soodnosne zaimke (prislove in prislovne izraze), ki jih klasična raba ni poznala; b) kopičenje znakov, ki natančneje določajo vrednost nekega veznika, bodisi da gre za splet soodnosnih zaimkov, ali pa za pojasnjevanje nekega izraza s pomočjo soodnosnice; c) najbolj pogostno soodnosnico, ilico, ki ji sledi prislov mox. bolj drzne.

Getica poznajo najvě̌je število odstopanj. Ni kršena samo klasična norma; konstrukcije so raznolike in

Jordanesova dela so neizmerljivega pomena za naše poznavanje pozne latinščine, tako ljudske kot kultivirane. Jordanesov tekst je pomemben tudi zato, ker se $\mathrm{v}$ njem pojavljajo značilnosti govorjenega jezika pa tudi črte pisarniškega stila, kar je pri avtorjih prejšnjih dob redko; redko celo pri piscih njegove dobe. Za osvetlitev naj navedemo: neponovljeni $u b i$ non s konjuktivom pluskvamperfeka; dum in mox s konjuktivom pluskvamperfekta; časovni quaetenus v pomenu 'do'. 